\title{
CLASS GROUPS OF INTEGRAL GROUP RINGS $\left({ }^{1}\right)$
}

BY

\section{REINER AND S. ULLOM}

ABSTRACT. Let $\Lambda$ be an $R$-order in a semisimple finite dimensional $K$-algebra, where $K$ is an algebraic number field, and $R$ is the ring of algebraic integers of $K$. Denote by $C(\Lambda)$ the reduced class group of the category of locally free left $\Lambda$-lattices. Choose $\Lambda=Z G$, the integral group ring of a finite group $G$, and let $\Lambda^{\prime}$ be a maximal $Z$-order in $Q G$ containing $\Lambda$. There is an epimorphism $C(\Lambda) \rightarrow C\left(\Lambda^{\prime}\right)$, given by $M \rightarrow \Lambda^{\prime} \otimes_{\Lambda} M$, for $M$ a locally free $\Lambda$-lattice. Let $D(\Lambda)$ be the kernel of this epimorphism; the groups $D(\Lambda), C(\Lambda)$ and $C\left(\Lambda^{\prime}\right)$ are all finite. Our main theorem is that $D(Z G)$ is a $p$-group whenever $G$ is a $p$-group. This generalizes Fröhlich's result for the case where $G$ is an abelian $p$-group. Our proof uses some facts about the center $F$ of $Q G$, as well as information about reduced norms. We also calculate $D(Z G)$ explicitly for $G$ cyclic of order $2 p$, dihedral of order $2 p$, or the quaternion group. In the se cases, the ring $Z G$ can be conveniently described by a pullback diagram.

1. Introduction. Let $R=\operatorname{alg}$ int $\{K\}$ be the ring of all algebraic integers in an algebraic number field $K$, and let $\Lambda$ be an $R$-order in a semisimple finite dimensional $K$-algebra $A$. By a $\Lambda$-lattice we mean always a left $\Lambda$-module which is finitely generated and torsionfree as $R$-module. For each maximal ideal $P$ of $R$, let $R_{P}$ denote the localization of $R$ at $P$, that is,

$$
R_{P}=\{\alpha / \beta: \alpha, \beta \in R, \beta \notin P\} .
$$

Set $\Delta_{P}=R_{P} \otimes_{R} \Lambda$, an $R_{P}$-order in $A$.

Two $\Lambda$-lattices $M, N$ are in the same genus if $M_{P} \cong N_{P}$ for each maximal ideal $P$ of $R$. We shall call $M$ locally free if $M$ is in the same genus as some free $\Lambda$-lattice $\Lambda^{(r)}$; here, $\Lambda^{(r)}$ denotes the direct sum of $r$ copies of $\Lambda$. In this case, we call $r$ the $\Lambda$-rank of $M$, and write $r=\operatorname{rank}_{\Lambda} M$. Of course, every locally free $\Lambda$-lattice is projective as left $\Lambda$-module.

We next define the (locally free) projective class group $P(\Lambda)$ as follows: let $\Re$ denote the free abelian group generated by symbols $[M]$, one for each isomorphism class of locally free $\Lambda$-lattices. Let $B_{0}$ be the subgroup of $\mathfrak{B}_{\text {generated by }}$ all expressions $[L \oplus M]-[L]-[M]$ where $L, M$ are locally free $\Lambda$-lattices. Then set $P(\Lambda)=\mathfrak{B}_{0} / \Re_{0}$, an abelian additive group. There is an additive epimorphism $P(\Lambda) \rightarrow Z$, induced by the mapping $[M] \rightarrow \operatorname{rank}_{\Lambda} M$; this epimorphism is split by the mapping $n \rightarrow \Lambda^{(n)}, n \in Z, n>0$. Let $C(\Lambda)$ be the kernel of this

Received by the editors July $1,1971$.

AMS 1970 subject classifications. Primary 16A54; Secondary 20C05, $20 \mathrm{C} 10$.

(1) This research was partially supported by a grant from the National Science Foundation. 
epimorphism, so $C(\Lambda)$ is the subgroup of $P(\Lambda)$ consisting of all expressions $[M]-[N]$, with $M, N$ locally free of the same $\Lambda$-rank. Clearly,

$$
P(\Lambda) \cong Z+C(\Lambda)
$$

as additive groups. For brevity, we refer to $C(\Lambda)$ as the class group of $\Lambda$. It is easily seen that $[M]-[N]=0$ in $C(\Lambda)$ if and only if $M$ and $N$ are stably isomorphic, that is, $M+\Lambda^{(s)} \cong N+\Lambda^{(s)}$ for some nonnegative integer $s$.

The methods of proof in Swan [13] show at once that every element of the class group $C(\Lambda)$ is expressible in the form $[J]-[\Lambda]$, where $J$ is a locally free left $\Lambda$-lattice in $A$. Since there are only a finite number of isomorphism classes of such J's (by the Jordan-Zassenhaus theorem), it follows that $C(\Lambda)$ is necessarily a finite group. Note further that $\left[J_{1}\right]-[\Lambda]=\left[J_{2}\right]-[\Lambda]$ in $C(\Lambda)$ if and only if $J_{1}$ and $J_{2}$ are stably isomorphic; that is, $J_{1} \dot{+} \Lambda^{(s)} \cong J_{2} \dot{+} \Lambda^{(s)}$ for some nonnegative integer $s$.

For the case where $\Lambda=R G$, the integral group ring of a finite group $G$, Swan [13] proved that every projective $\Lambda$-lattice is locally free. Thus our $C(\Lambda)$ is the usual "reduced projective class group."

On the other hand, let $\Lambda^{\prime}$ be a maximal $R$-order in a semisimple $K$-algebra $A$. A projective $\Lambda^{\prime}$-lattice $M^{\prime}$ is called special if $K \otimes_{R} M^{\prime}$ is $A$-free. From the theory of maximal orders (see [4], [12] or [15]), we know that every $\Lambda^{\prime}$-lattice $M^{\prime}$ is projective, and that $M^{\prime}$ is locally free if and only if $M^{\prime}$ is special. Thus our $C\left(\Lambda^{\prime}\right)$ is the usual "reduced special projective class group." Since most of our calculations below depend on working with locally free lattices, it seems desirable to single out this class of projective lattices rather than the class of special projectives.

Suppose now that $\Lambda^{\prime}$ is a maximal $R$-order in $A$ containing $\Lambda$. There is then an additive homomorphism $P(\Lambda) \rightarrow P\left(\Lambda^{\prime}\right)$, induced by letting

$$
[M] \rightarrow\left[\Lambda^{\prime} \otimes_{\Lambda} M\right], \quad M=\text { locally free } \Lambda \text {-lattice. }
$$

This homomorphism induces a homomorphism $\eta: C(\Lambda) \rightarrow C\left(\Lambda^{\prime}\right)$, and Swan [14] showed that $\eta$ is in fact an epimorphism. Let $D(\Lambda)$ be the kernel of $\eta$, so there is an exact sequence of additive groups

$$
0 \rightarrow D(\Lambda) \rightarrow C(\Lambda) \rightarrow C\left(\Lambda^{\prime}\right) \rightarrow 0,
$$

and obviously

$$
|C(\Lambda)|=|D(\Lambda)| \cdot\left|C\left(\Lambda^{\prime}\right)\right| \cdot
$$

The class group $C\left(\Lambda^{\prime}\right)$ of the maximal order $\Lambda^{\prime}$ is easily determined, and hence we can find the order of $C(\Lambda)$ by calculating that of $D(\Lambda)$. In this direction, Fröhlich [8] has shown that $|D(Z G)|$ is a power of $p$ whenever $G$ is an abelian $p$-group. One of the main results of the present article is the fact that $|D(Z G)|$ is a power of $p$, for an arbitrary finite $p$-group $G$, not necessarily abelian. We may remark that this conclusion need not hold, even for abelian $p$-groups, when $Z$ is replaced by some larger ring of algebraic integers. This 
already follows from the work of Ullom [16].

We have also included explicit calculations of $C(Z G)$ and $D(Z G)$ for the following groups $G$ : cyclic group of order $2 p$, dihedral group of order $2 p$, quaternion group of order 8 . Here, $p$ is any odd prime. Some of these calculations could be simplified slightly by using Milnor's Mayer-Vietoris sequence in K-theory (see Bass [1]). We have avoided using this sequence, however, in order to illustrate the computational aspects of our present approach. A later work [18] will be devoted to $K$-theory calculations.

The following notation will be used throughout:

$\mathrm{rad}=$ Jacobson radical.

$(R)^{n \times n}=$ ring of all $n \times n$ matrices over a ring $R$.

$M^{(k)}=$ direct sum of $k$ copies of $M$.

$R_{\hat{P}}=P$-adic completion of the integral domain $R$ at a prime $P$ of the quotient field of $R$.

$\Sigma, \dot{+}$ : direct sum.

$\operatorname{ann}_{R} X=\{\alpha \in R: \alpha X=0\}=R$-annihilator of the $R$-module $X$.

$u(\Lambda)=$ group of units $(2)$ of the ring $\Lambda$.

General references for this paper are [11], [12] and [15]. The authors wish to thank Professor A. Fröhlich for some helpful conversations, and for pointing out Lemma 3.4 below.

2. Addition in the class group. The purpose of this section is to review some well-known results for the convenience of the reader; proofs may be found in the general references cited at the end of $\$ 1$. As before, we let $\Lambda$ be any $R$-order in the semisimple $K$-algebra $A$.

Suppose that $M$ is a locally free $\Lambda$-lattice of $\Lambda$-rank $r$. It follows readily from the approximation theorem for algebraic numbers that, given any nonzero ideal $q$ of $R$, there exists a $\Lambda$-embedding $M \rightarrow \Lambda^{(r)}$ such that

$$
\operatorname{ann}_{R}\left(\Lambda^{(r)} / M\right)+q=R \text {. }
$$

The methods of proof in Swan [13] then show that there exists a locally free left $\Lambda$-lattice $J$ in $A$ such that $M \cong \Lambda^{(r-1)}+J$. Hence every element of the (locally free) class group $C(\Lambda)$ is expressible in the form

$$
x_{j}=[J]-[\Lambda], \quad J=\text { locally free } \Lambda \text {-free lattice in } A .
$$

As pointed out in $\S 1$, we have $x_{J_{1}}=x_{J_{2}}$ if and only if $J_{1}$ and $J_{2}$ are stably isomorphic.

How can we calculate $x_{J_{1}}+x_{J_{2}}$ in $C(\Lambda)$ ? First of all, embed $J_{1}$ in $\Lambda$ so as to get a $\Lambda$-exact sequence

(2) A unit of $\mathbf{\Lambda}$ is an element of $\mathbf{\Lambda}$ which has a two-sided inverse in $\Lambda$. 


$$
0 \rightarrow J_{1} \stackrel{i_{1}}{\longrightarrow} \Lambda \rightarrow T_{1} \rightarrow 0,
$$

with $T_{1}$ an $R$-torsion $\Lambda$-module. Then choose a $\Lambda$-exact sequence

$$
0 \rightarrow J_{2} \stackrel{i_{2}}{\longrightarrow} \Lambda \rightarrow T_{2} \rightarrow 0
$$

with $T_{2}$ an $R$-torsion $\Lambda$-module such that

$$
\operatorname{ann}_{R} T_{1}+\operatorname{ann}_{R} T_{2}=R \text {. }
$$

It follows easily that the map $\left(i_{1}, i_{2}\right): J_{1}+J_{2} \rightarrow \Lambda$ is a $\Lambda$-epimorphism, and hence that

$$
J_{1}+J_{2} \cong \Lambda+J_{3}
$$

for some $\Lambda$-lattice $J_{3}$ in $A$. We may conclude from this that for each maximal ideal $P$ of $R$,

$$
\Lambda_{\hat{p}} \dot{+} \Lambda_{\hat{p}} \cong \Lambda_{\hat{p}} \dot{+}\left(J_{3}\right)_{\hat{p}}
$$

where the subscript $\hat{P}$ indicates passage to $P$-adic completions. Since the KrullSchmidt theorem is valid for $\Lambda_{\hat{P}}$-modules, the above isomorphism implies that $\Lambda_{\hat{p}} \cong\left(J_{3}\right)_{\hat{P}}$. This in turn implies that $\Lambda_{P} \cong\left(J_{3}\right)_{P}$, so we have verified that $J_{3}$ is also locally free. The operation of addition in $C(\Lambda)$ is then given by the formula: $x_{J_{1}}+x_{J_{2}}=x_{J_{3}}$.

For the remainder of this section, suppose that $A$ is commutative. Let us show that the $\Lambda$-lattice $J_{3}$ occurring in (2.4) is such that

$$
J_{3} \cong J_{1} \cdot J_{2} \quad(=\text { product of } \Lambda \text {-ideals in } A) \text {. }
$$

Indeed, from (2.1) we obtain an exact sequence of $\Lambda$-modules

$$
0 \rightarrow J_{1} \otimes J_{2} \rightarrow J_{2} \rightarrow T_{1} \otimes J_{2} \rightarrow 0
$$

where $\otimes$ means $\otimes_{\Lambda}$. On the other hand, from (2.2) we get an exact sequençe

$$
\operatorname{Tor}_{1}^{\Lambda}\left(T_{1}, T_{2}\right) \rightarrow T_{1} \otimes J_{2} \rightarrow T_{1} \rightarrow T_{1} \otimes T_{2} \quad(\rightarrow 0) \text {. }
$$

By virtue of (2.3), the first and last terms of the above sequence vanish, and thus $T_{1} \otimes J_{2} \cong T_{1}$. Consequently there is an exact sequence

$$
0 \rightarrow J_{1} \otimes J_{2} \rightarrow J_{2} \rightarrow T_{1} \rightarrow 0 \text {. }
$$

Applying Schanuel's lemma to the pair of sequences (2.1) and (2.5), we obtain

$$
J_{1}+J_{2} \cong \Lambda+\left(J_{1} \otimes J_{2}\right) \text {. }
$$

Since the map $\xi \otimes \eta \rightarrow \xi \eta$ gives an isomorphism $J_{1} \otimes J_{2} \cong J_{1} \cdot J_{2}$, we may conclude that

$$
J_{1}+J_{2} \cong \Lambda+J_{1} J_{2} \text { and } x_{J_{1}}+x_{J_{2}}=x_{J_{1} J_{2}} \text { in } C(\Lambda)
$$

On the other hand, since $A$ is commutative, we may define an ideal class group $\bar{C}(\Lambda)$ in a more natural manner, as follows: Relative to the usual multiplication of $\Lambda$-ideals in $A$, the locally free $\Lambda$-lattices( 3 ) in $A$ form a multiplicative group $\bar{I}(\Lambda)$. The set of all principal ideals

(3) Note that every locally free $\Lambda$-lattice in $A$ is invertible. 
$\{\Lambda x: x \in A, x$ invertible in $A\}$

forms a subgroup $\bar{I}_{0}(\Lambda)$ of $\bar{I}(\Lambda)$. We now set

$$
\bar{C}(\Lambda)=\bar{I}(\Lambda) / \bar{I}_{0}(\Lambda)
$$

the multiplicative group of classes of locally free $\Lambda$-ideals in $A$. (This group $\bar{C}(\Lambda)$ is the same as $\operatorname{Pic}(\Lambda)$; see Bass [1].)

Let us show that the multiplicative group $\bar{C}(\Lambda)$ is isomorphic to the additive group $C(\Lambda)$. Define a map $\mu: \bar{C}(\Lambda) \rightarrow C(\Lambda)$ by

$$
\mu(\text { ideal class of } J)=[J]-[\Lambda],
$$

where $J$ is any locally free left $\Lambda$-lattice in $A$. The definition is meaningful, since if $J_{1}$ is in the same ideal class as $J_{2}$, then $J_{1} \cong J_{2}$ as left $\Lambda$-modules, and thus

$$
\left[J_{1}\right]-[\Lambda]=\left[J_{2}\right]-[\Lambda] \text { in } C(\Lambda) .
$$

We have already remarked at the beginning of this section that $\mu$ is epic. We claim that $\mu$ is one-to-one. For if $[J]-[\Lambda]=0$ in $C(\Lambda)$, then

$$
J+\Lambda^{(r)} \cong \Lambda+\Lambda^{(r)}
$$

for some nonnegative integer $r$. But the $(r+1)$ st exterior power (over $\Lambda$ ) of $J+\Lambda^{(r)}$ is $J$ itself. Hence (2.7) implies that $J \cong \Lambda$, that is, $J \in \bar{I}_{0}(\Lambda)$. This completes the proof that $\mu$ gives a one-to-one mapping of $\bar{C}(\Lambda)$ onto $C(\Lambda)$. Equations (2.6) show that $\mu$ is a group homomorphism, so we have established the isomorphism $C(\Lambda) \cong \bar{C}(\Lambda)$.

In a forthcoming article [17], Fröhlich has considered the group $\mathrm{Pic}(Z G)$ for non-abelian $G$. The authors wish to thank Professor Fröhlich for the opportunity of reading a preliminary version of this article. The relationship between $\operatorname{Pic}(Z G)$ and our $C(Z G)$ will be investigated in a future work [19].

3. Explicit formulas for the class group. Returning to the general case, we fix the following notation for the remainder of this article. Let $\Lambda$ be any $R$-order in the semisimple $K$-algebra $A$, and let $\Lambda^{\prime}$ be some maximal $R$-order in $A$ containing $\Lambda$. We may write

$$
A=\sum_{i=1}^{m} A_{i} \quad \text { (simple components) }
$$

$$
\begin{aligned}
\Lambda^{\prime} & =\sum_{i=1}^{m} \cdot \Lambda_{i}, \quad \Lambda_{i}=\text { maximal } R \text {-order in } A_{i}, \\
F & =\sum_{i=1}^{m} K_{i}, \quad K_{i}=\text { center of } A_{i}, \quad C=\sum_{i=1}^{m} R_{i}, \quad R_{i}=\operatorname{alg} \text { int }\left\{K_{i}\right\} \subset \Lambda_{i} .
\end{aligned}
$$


Each $\Lambda_{i}$ is then a maximal $R_{i}$-order in the $i$ th simple component $A_{i}$ of $A$. Further, $F$ is the center of $A$, and $C$ is the unique maximal $R$-order in $F$.

(i) Reduced norms. Let $N_{i}: A_{i} \rightarrow K_{i}$ be the reduced norm map. If $A_{i}$ happens to be a full matrix algebra $\left(K_{i}\right)^{\frac{m_{i} \times m_{i}}{i}}$ over the field $K_{i}$, and if we let the element $x \in A_{i}$ map onto the $m_{i} \times m_{i}$ matrix $\mathbf{x}$ over $K_{i}$, then $N_{i}(x)=\operatorname{det} \mathbf{x}$. In the general case (see [2]), the reduced norm $N_{i}$ is obtained by first passing to a splitting field for $A_{i}$, and then using the preceding comment.

Now define the reduced norm map $N: A \rightarrow F$ by working in each simple component separately, that is, $N=\Sigma \cdot N_{i}$.

In order to describe the image $N_{i}\left(A_{i}\right)$ in $K_{i}$, we proceed as follows. For $P$ any prime of $K_{i}$ (finite or infinite), let $\left(K_{i}\right)_{\hat{P}}$ denote the $P$-adic completion of $K_{i}$. Set

$$
\left(A_{i \hat{p}}=\left(K_{i}\right)_{\hat{p}} \otimes_{K_{i}} A_{i},\right.
$$

a simple algebra with center $\left(K_{i}\right)_{\hat{P}}$. We shall say that $P$ ramifies in $A_{i}$ if $\left(A_{i}\right)_{\hat{P}}$ is not a full matrix algebra over the field $\left(K_{i}\right)_{\hat{P}}$. A proof of the following result of Hasse may be found in Swan-Evans [15, Theorem 7.6]:

(3.2) Theorem. A nonzero element $a \in K_{i}$ lies in $N_{i}\left(A_{i}\right)$ if and only if $\alpha_{\hat{P}}>0$ for every real infinite prime $P$ of $K_{i}$ which ramifies in $A_{i}$. Here, $\alpha_{\hat{P}}$ denotes the image of $\alpha$ in $\left(K_{i}\right) \hat{p}$.

(ii) The Eichler condition. Call $A_{i}$ a totally definite quaternion algebra (over its center $K_{i}$ ) if every infinite prime $P$ of $K_{i}$ is real, and for every such $P$ the algebra $\left(A_{i}\right)_{\hat{P}}$ is a quaternion skewfield over its center $\left(K_{i}\right)_{\hat{P}}$.

We shall say that the algebra $A$ satisfies the Eichler condition if no simple component $A_{i}$ of $A$ is a totally definite quaternion algebra. Commutative algebras automatically satisfy the Eichler condition.

(iii) Localization. Let $f$ be a nonzero ideal of $R$ (eventually to be chosen so that $\left.F \cdot \Lambda^{\prime} \subset \Lambda\right)$. Call an element $\alpha \in F$ prime to $F$ if, for each prime ideal $P$ of $R$ dividing $F, \alpha$ is a unit in the localization $C_{P}$. Rather than use idèles, we introduce the semilocal ring

$$
R_{F}=\bigcap_{P \text { divides }} R_{P}=\{\alpha / \beta: \alpha, \beta \in R, \beta \text { prime to } f\} .
$$

This ring is a principal ideal domain, with maximal ideals $\left\{P \cdot R_{F}: P\right.$ divides $\left.f\right\}$. Now set

$$
\Lambda_{\mathrm{F}}=R_{\mathrm{F}} \otimes_{R} \Lambda, \quad C_{\mathrm{F}}=R_{\mathrm{F}} \otimes_{R} C,
$$

and so on. It is then clear that an element $\alpha \in F$ is prime to $F$ if and only if $a \in u\left(C_{F}\right)$, the group of units of $C_{F}$.

Likewise, an element $x \in \Lambda$ is said to be prime to $F$ if $x \in u\left(\Lambda_{F}\right)$, that is, if $\Lambda_{f}=\Lambda_{f} \cdot x$. In this case, it follows that $\Lambda_{f}^{\prime}=\Lambda_{f}^{\prime} \cdot x$, so that $x$ is prime to $f$ when we view $x$ as an element of the larger order $\Lambda^{\prime}$. The converse is also true, however: 
(3.3) Lemma. Let $x \in \Lambda \subset \Lambda^{\prime}$, and suppose that $x$ is a unit in $\Lambda_{F}^{\prime}$. Then $x$ is also a unit in $\Lambda_{\mathrm{F}}$. Hence the property of being "prime to $f$ " is independent of the choice of R-order.

This lemma is an immediate consequence of the following more general fact:

(3.4) Lemma. Let $\Lambda \subset \Lambda^{\prime}$ be R-orders in A. Then $\Lambda \cap u\left(\Lambda^{\prime}\right)=u(\Lambda)$.

Proof. Let $x \in \Lambda$; we must show that $x \in u(\Lambda)$ if and only if $x \in u\left(\Lambda^{\prime}\right)$. Now $x \in u(\Lambda)$ if and only if $\Lambda=\Lambda x$, so we need to prove that $\Lambda=\Lambda x$ if and only if $\Lambda^{\prime}=\Lambda^{\prime} x$. Let $[\Lambda: \Lambda x]$ denote the order ideal of the $R$-torsion $\Lambda$-module $\Lambda / \Lambda x$, that is, $[\Lambda: \Lambda x]$ is the product of the $R$-annihilators of the $R$-composition factors of $\Lambda / \Lambda x$ (see [3] or [7]). Relative to a $K$-basis of $A$, right multiplication by $x$ acting on $A$ gives rise to a matrix $\mathbf{M}(x)$, and we have

$$
[\Lambda: \Lambda x]=R \cdot \operatorname{det} \mathbf{M}(x) \text {. }
$$

Since the same equation holds with $\Lambda$ replaced by $\Lambda^{\prime}$, we obtain $[\Lambda: \Lambda x]=$ $\left[\Lambda^{\prime}: \Lambda^{\prime} x\right]$. This implies the desired result.

Remark. Using the notation of the preceding proof, we may observe that every prime ideal of $R$ which divides $C \cdot N(x)$ also divides $R \cdot \operatorname{det} \mathbf{M}(x)$, and conversely: Hence an element $x \in \Lambda$ is a unit in $\Lambda$ if and only if $N(x)$ is a unit in $R$.

Now let $u\left(\Lambda_{F}\right)$ be the group of units of the ring $\Lambda_{F}$. Obviously

$$
\{x \in \Lambda: x \text { prime to } f\} \subset u\left(\Lambda_{f}\right) \text {, }
$$

by definition of "prime to $F$ ". On the other hand, any element $y \in u\left(\Lambda_{\digamma}\right)$ is expressible as $y=x / r$, with $x \in \Lambda, r \in R$, where $r$ is prime to $f$. Then both $x$ and $r$ are elements of $\Lambda$ prime to $F$. This shows that $u\left(\Lambda_{F}\right)$ is the multiplicative group generated by the set

$$
\{x \in \Lambda: x \text { prime to } f\} \text {. }
$$

Furthermore, the preceding remark shows that an element $x \in \Lambda$ is prime to $f$ if and only if its reduced norm $N(x)$ is prime to $F$.

(iv) Explicit formulas when Eichler condition holds. Hereafter, let $f$ be a nonzero ideal of $R$ such that $F \cdot \Lambda^{\prime} \subset \Lambda$, where $\Lambda^{\prime}$ is a maximal $R$-order in $A$ containing $\Lambda$. Such an ideal $F$ always exists. (For example, when $\Lambda$ is the integral group ring $R G$ of a finite group $G$, we may choose $F=|G| \cdot R$.) Denote by $I(C, F)$ the multiplicative group of all $C$-ideals in $F$, nonzero at each component, which are prime to $f$.

Let $I(\Lambda)$ be the subgroup of $I(C, F)$ generated by all ideals

$$
\{C \cdot N(x): x \in \Lambda, x \text { prime to } F\} \text {. }
$$

Since $(C \cdot N(x)) \cdot(C \cdot N(y))=C \cdot N(x y)$ for $x, y \in \Lambda$ prime to $F$, and since the set of elements $\{x \in \Lambda: x$ prime to $F\}$ generates the group $u\left(\Lambda_{F}\right)$, we conclude at once that 


$$
I(\Lambda)=\left\{C \cdot N(x): x \in u\left(\Lambda_{F}\right)\right\}
$$

Likewise, we set

$$
I\left(\Lambda^{\prime}\right)=\left\{C \cdot N(y): y \in u\left(\Lambda_{F}^{\prime}\right)\right\} .
$$

We now quote the following basic result due to Jacobinski [9]:

(3.7) Theorem. If the algebra A satisfies the Eicbler condition, then there is a commutative diagram

$$
\begin{aligned}
& C(\Lambda) \cong I(C, F) / I(\Lambda) \\
& \left.\eta \downarrow \quad \eta^{\prime}\right\rfloor \\
& C\left(\Lambda^{\prime}\right) \cong I(C, F) / I\left(\Lambda^{\prime}\right)
\end{aligned}
$$

where $\eta$ is defined by applying $\Lambda^{\prime} \otimes_{\mathbf{\Lambda}} \cdot$ to $\Lambda$-lattices, and $\eta^{\prime}$ is the natural epimorphism. Consequently,

$$
D(\Lambda)=\operatorname{ker} \eta \cong I\left(\Lambda^{\prime}\right) / I(\Lambda) .
$$

Remark. The groups $I\left(\Lambda^{\prime}\right)$ and $I(\Lambda)$ depend on $F$. However, Jacobinski showed that (up to isomorphism) the groups $C(\Lambda), C\left(\Lambda^{\prime}\right)$ and $D(\Lambda)$ are independent of the choice of the maximal order $\Lambda^{\prime}$ containing $\Lambda$, and are also independent of the choice of the nonzero ideal $F$ of $R$ such that $F \cdot \Lambda^{\prime} \subset \Lambda$.

Let us now define a homomorphism

$$
\theta: u\left(\Lambda_{F}^{\prime}\right) \rightarrow I\left(\Lambda^{\prime}\right)
$$

by setting $\theta(x)=C \cdot N(x), x \in u\left(\Lambda^{\prime}{ }_{f}\right)$. Then $\theta$ is an epimorphism with kernel

$$
\left\{x \in u\left(\Lambda_{F}^{\prime}\right): N(x)=\text { unit in } C\right\} \text {. }
$$

Denote by $C^{*}$ the group of units of $C$, and set

$$
N^{-1}\left(C^{*}\right)=\left\{x \in A: N(x) \in C^{*}\right\}
$$

Then

$$
\operatorname{ker} \theta=u\left(\Lambda_{F}^{\prime}\right) \cap N^{-1}\left(C^{*}\right),
$$

and therefore

$$
u\left(\Lambda_{F}^{\prime}\right) /\left\{u\left(\Lambda_{F}^{\prime}\right) \cap N^{-1}\left(C^{*}\right)\right\} \cong I\left(\Lambda^{\prime}\right) .
$$

However, $N^{-1}\left(C^{*}\right) \triangleleft u\left(\Lambda_{f}^{\prime}\right) \cdot N^{-1}\left(C^{*}\right)$, since $N^{-1}\left(C^{*}\right)$ contains the commutator subgroup of $u\left(\Lambda^{\prime}{ }_{f}\right)$. We thus may deduce from the above isomorphism the fact that

$$
\left(u\left(\Lambda_{F}^{\prime}\right) \cdot N^{-1}\left(C^{*}\right)\right) / N^{-1}\left(C^{*}\right) \cong I\left(\Lambda^{\prime}\right),
$$

where the isomorphism is induced by letting an element $x$ in the numerator map onto $C \cdot N(x)$. 
In the same manner, we obtain

$$
\left(u\left(\Lambda_{F}\right) \cdot N^{-1}\left(C^{*}\right)\right) / N^{-1}\left(C^{*}\right) \cong I(\Lambda)
$$

Consequently, we have

$$
D(\Lambda) \cong \frac{I\left(\Lambda^{\prime}\right)}{I(\Lambda)} \cong \frac{u\left(\Lambda_{F}^{\prime}\right) \cdot N^{-1}\left(C^{*}\right)}{u\left(\Lambda_{\digamma}\right) \cdot N^{-1}\left(C^{*}\right)} \cong \frac{u\left(\Lambda_{F}^{\prime}\right)}{u\left(\Lambda_{\digamma}^{\prime}\right) \cap\left\{u\left(\Lambda_{\digamma}\right) \cdot N^{-1}\left(C^{*}\right)\right\}} .
$$

Note that $u\left(\Lambda_{f}\right) \cdot N^{-1}\left(C^{*}\right) \triangleleft u\left(\Lambda^{\prime} f\right) N^{-1}\left(C^{*}\right)$, since $N^{-1}\left(C^{*}\right)$ contains all commutators. Formula (3.8) avoids the use of reduced norms, except for the calculation of $N^{-1}\left(C^{*}\right)$.

We conclude this subsection by giving Jacobinski's construction for the element $\delta_{x} \in D(\Lambda)$ which corresponds to an element $x \in u\left(\Lambda_{f}^{\prime}\right)$ in the isomorphism (3.8). Namely, write $x=y z^{-1}$ with both $y$ and $z$ in $\Lambda^{\prime}$ and prime to $F$. Then $\delta_{x}=\left[\Lambda \cap \Lambda^{\prime} y\right]-\left[\Lambda \cap \Lambda^{\prime} z\right]$ is the desired element of $D(\Lambda)$. (It is easily checked that both $\Lambda \cap \Lambda^{\prime} y$ and $\Lambda \cap \Lambda^{\prime} z$ are locally free $\Lambda$-lattices, and that $\delta_{x}$ lies in the kernel of the mapping $C(\Lambda) \rightarrow C\left(\Lambda^{\prime}\right)$.)

(v) Explicit formulas in the general case. We now turn to the case where $A$ need not satisfy the Eichler condition. Let us form

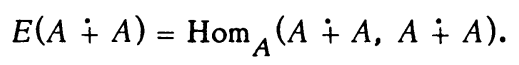

Suppose that the $i$ th simple component $A_{i}$ of $A$ is given by $A_{i} \cong\left(D_{i}\right)^{m_{i} \times m_{i}}$, $D_{i}=$ skewfield with center $K_{i}$. Then

$$
E(A+A) \cong \sum_{i=1}^{m} \cdot\left(D_{i}\right)^{2 m_{i} \times 2 m_{i}}
$$

Thus $E(A+A)$ has the same center $F$ as $A$, and is a semisimple $K$-algebra which automatically satisfies the Eichler condition. Furthermore,

$$
E\left(\Lambda^{\prime}+\Lambda^{\prime}\right)=\operatorname{Hom}_{\Lambda^{\prime}}\left(\Lambda^{\prime}+\Lambda^{\prime}, \Lambda^{\prime}+\Lambda^{\prime}\right)
$$

is a maximal $R$-order in $E(A+A)$.

Let $N^{*}: E(A+A) \rightarrow F$ be the reduced norm map. We shall say that an element $x \in E(\Lambda+\Lambda)$ is prime to $F$ if $x$ is a unit in $\{E(\Lambda+\Lambda)\}_{F}$, or equivalently, if $N^{*}(x)$ is prime to $F$. There is an obvious ring isomorphism

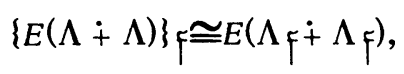

and we shall always identify these rings with one another. As before, let $I(C, f)$ be the group of all $C$-ideals of $F$ prime to $F$, and let $J(\Lambda)$ be the subgroup of $I(C, F)$ generated by the set of all principal ideals

$$
\left\{C \cdot N^{*}(y): y \in E(\Lambda+\Lambda), y \text { prime to } F\right\} \text {. }
$$

As in the preceding section, we obtain 


$$
J(\Lambda)=\left\{C \cdot N^{*}(y): y=\text { unit in } E\left(\Lambda_{\mathcal{F}}+\Lambda_{\digamma}\right)\right\} .
$$

Likewise, we set

$$
J\left(\Lambda^{\prime}\right)=\left\{C \cdot N^{*}(y): y=\text { unit in } E\left(\Lambda_{F}^{\prime}+\Lambda_{F}^{\prime}\right)\right\} .
$$

The following basic result is due to Jacobinski [9]:

(3.11) Theorem. Whether or not A satisfies the Eicbler condition, there is a commutative diagram

$$
\begin{gathered}
C(\Lambda) \cong I(C, f) / J(\Lambda) \\
\eta \downarrow \\
C\left(\Lambda^{\prime}\right) \cong I(C, f) / J\left(\Lambda^{\prime}\right)
\end{gathered}
$$

where $\eta$ is defined by applying $\Lambda^{\prime} \otimes_{\Lambda} \quad$ to $\Lambda$-lattices, and $\eta^{\prime \prime}$ is the natural epimorphism. Consequently,

$$
D(\Lambda)=\operatorname{ker} \eta \cong J\left(\Lambda^{\prime}\right) / J(\Lambda)
$$

Up to isomorphism, these formulas are independent of the choice of maximal order $\Lambda^{\prime}$ containing $\Lambda$, and of the ideal $F$ of $R$ such that $F \cdot \Lambda^{\prime} \subset \Lambda$. Furthermore, when $A$ satisfies the Eichler condition, we have

$$
J\left(\Lambda^{\prime}\right)=I\left(\Lambda^{\prime}\right), \quad J(\Lambda)=I(\Lambda)
$$

There are obvious analogues of formula (3.8), which we shall not write down explicitly.

4. Functorial property of class groups. We intend to show that any group epimorphism $G \rightarrow \bar{G}$ induces epimorphisms (of additive groups)

$$
C(R G) \rightarrow C(R \bar{G}), \quad D(R G) \longrightarrow D(R \bar{G}) .
$$

This will be a consequence of some fairly general considerations, together with the formulas given in $\$ 3$. Let $\Lambda$ be any $R$-order in the semisimple algebra $A$, let $\Lambda^{\prime}$ be a maximal $R$-order in $A$ containing $\Lambda$, and let $f$ be a nonzero ideal of $R$ such that $F \cdot \Lambda^{\prime} \subset \Lambda$. Let $A \rightarrow \bar{A}$ be an epimorphism of $K$-algebras, and let $\Lambda$ map onto the $R$-order $\bar{\Lambda}$. We shall show that there are epimorphisms

$$
C(\Lambda) \rightarrow C(\bar{\Lambda}), \quad D(\Lambda) \rightarrow D(\bar{\Lambda})
$$

which of course imply the analogous results for the special case in (4.1). For convenience, we treat only the case where $A$ satisfies the Eichler condition, since the proof in the general case proceeds in an entirely similar manner.

The kernel of the map $A \rightarrow \bar{A}$ is a two-sided ideal in $A$, hence is a direct sum of some of the simple components of $A$. This kernel is therefore expressible in the form $A(1-e)$, where $e$ is some central idempotent in $A$. Then $e$ maps onto $\bar{e} \in \bar{A}$, where $\bar{e}$ is the identity element of $\bar{A}$, and we have $A=A e \oplus A(1-e)$, 
$A e \cong \bar{A}, \Lambda e \cong \bar{\Lambda}$. Letting $C$ again denote the maximal $R$-order in the center $F$ of $A$, it is clear that $e \in C$, and thus $C=C e \oplus C(1-e)$.

Let $N^{\prime}, N^{\prime \prime}$ be reduced norm maps, where

$$
N^{\prime}: A e \rightarrow F e, \quad N^{\prime \prime}: A(1-e) \rightarrow F(1-e) .
$$

Then $N=N^{\prime}+N^{\prime \prime}$; that is,

$$
N(x)=N^{\prime}(x e)+N^{\prime \prime}(x(1-e)), \quad x \in A .
$$

There is an obvious map from the group of $C$-ideals in $F$ onto the group of $\mathrm{Ce}$-ideals in $\mathrm{Fe}$, given by $a \rightarrow a e$. This map induces an epimorphism.

$$
\tau: I(C, F) \rightarrow I(C e, F) \text {. }
$$

If $x \in u\left(\Lambda_{F}\right)$, then $x y=1$ for some $y \in u\left(\Lambda_{\mathcal{F}}\right)$. Therefore, $x e \cdot y e=e$, so $x e \epsilon$ $u\left(\Lambda_{\mathcal{F}} \cdot e\right)$. Furthermore, for $x \in u\left(\Lambda_{\mathcal{F}}\right)$, we obtain

$$
\tau\{C \cdot N(x)\}=C e \cdot N(x)=C e \cdot N^{\prime}(x e) .
$$

This implies at once that $\tau$ maps $I(\Lambda)$ into $I(\Lambda e)$, where

$$
I(\Lambda)=\left\{C \cdot N(x): x \in u\left(\Lambda_{\digamma}\right)\right\}, \quad I(\Lambda e)=\left\{C e \cdot N^{\prime}(z): z \in u\left(\Lambda_{\digamma} \cdot e\right)\right\} .
$$

Hence $\tau$ induces an epimorphism

$$
I(C, F) / I(\Lambda) \rightarrow I(C e, F) / I(\Lambda e) .
$$

Since $\Lambda e \cong \bar{\Lambda}$, we deduce from Theorem 3.7 that there is an epimorphism $C(\Lambda)$ $\rightarrow C(\bar{\Lambda})$, as claimed.

Next we note that $e \in \Lambda^{\prime}$, and therefore $\Lambda^{\prime}=\Lambda^{\prime} e \oplus \Lambda^{\prime}(1-e)$. This shows that $C\left(\Lambda^{\prime}\right)=C\left(\Lambda^{\prime} e\right) \oplus C\left(\Lambda^{\prime}(1-e)\right)$, so there is also an epimorphism $C\left(\Lambda^{\prime}\right) \rightarrow$ $C\left(\Lambda^{\prime} e\right)$.

It remains for us to prove the existence of an epimorphism $D(\Lambda) \rightarrow D(\bar{\Lambda})$. Now $\Lambda^{\prime} e$ is a maximal order in $A e$ containing $\Lambda e$, and

$$
I\left(\Lambda^{\prime} e\right)=\left\{C e \cdot N^{\prime}(z): z=\text { unit in } \Lambda^{\prime} f \cdot e\right\} .
$$

Given any unit $z \in \Lambda^{\prime} F \cdot e$, we may find an element $x \in u\left(\Lambda_{f}^{\prime}\right)$ such that $z=x e$. But then formula (4.3) shows that $\mathrm{Ce} \cdot N^{\prime}(z)$ lies in the image of $\tau$. This proves that $\tau$ maps $I\left(\Lambda^{\prime}\right)$ onto $I\left(\Lambda^{\prime} e\right)$. Therefore $\tau$ induces an epimorphism

$$
I\left(\Lambda^{\prime}\right) / I(\Lambda) \rightarrow I\left(\Lambda^{\prime} e\right) / I(\Lambda e)
$$

so we have shown the existence of an epimorphism $D(\Lambda) \rightarrow D(\bar{\Lambda})$, as desired.

5. Odd $p$-groups. Let $G$ be a finite $p$-group, where $p$ is any prime, and set $A=Q G, \Lambda=Z G$. Keep the notation of $\$ 3$, especially that listed in (3.1). We may choose the ideal $\dot{F}=|G| \cdot Z$, and hence we may write "prime to $p$ " in place of 
"prime to f" throughout. The discussion splits into two cases, depending on wherher or not $p$ is odd. We shall consider first the easier case where $p$ is odd.

We begin by recalling some preliminary results from representation theory and algebraic number theory.

(5.1) Theorem. Let $G$ be an odd p-group, and let $K_{i}$ denote the center of the ith simple component of $Q G$. Then for each $i$, the field $K_{i}$ is a cyclotomic field $Q(\omega)$ for some $p^{n}$ th root of unity $\omega$. There is exactly one simple component, say $A_{1}$, for which $K_{1}=Q$; in fact, $A_{1}=K_{1}=Q$. Furthermore, each $A_{i}$ is a full matrix algebra over $K_{i}$.

Reference. Feit [6, (14.5)]; the result is due to Witt and Roquette.

(5.2) Theorem. Let $K_{i}=Q(\omega)$, where $\omega$ is a primitive $p^{n}$ th root of unity, with $n \geq 1$. Let $R_{i}=$ alg int $\left\{K_{i}\right\}$. Then there is a unique prime ideal $P_{i}$ of $R_{i}$ containing $p$, and $R_{i} / P_{i} \cong Z / p Z$. Given any element $\alpha \in R_{i}$ prime to $P_{i}$, there exists an element $\beta \in R_{i}$ such that $\beta \equiv 1\left(\bmod P_{i}\right)$, and $R_{i} \cdot \alpha=R_{i} \cdot \beta$.

Proof. The uniqueness of $P_{i}$, and the fact that $R_{i} / P_{i} \cong Z / p Z$, both follow from the well-known result that $p$ is completely ramified in $K_{i}$. Now let $a \in R_{i}$ be prime to $P_{i} ;$ then we can choose $m \in Z$ such that $\alpha \equiv m\left(\bmod P_{i}\right)$, and clearly $p \nmid m$. Set $u=\left(\omega^{m}-1\right) /(\omega-1)$. Then $u$ is a unit in $R_{i}$, and $u \equiv$ $m\left(\bmod P_{i}\right)$ since $P_{i}=(1-\omega) R_{i}$. The element $\beta=u^{-1} \alpha$ satisfies the desired conditions.

It is clear from (5.1) that $A$ satisfies the Eichler condition, and that for each $i$, no infinite prime of $K_{i}$ ramifies in $A_{i}$. As in $\$ 3$, let $I(C, p)$ be the group of all $C$-ideals in $F$ which are prime to $p$, and set

$$
I\left(\Lambda^{\prime}\right)=\left\{C \cdot N(x): x \in u\left(\Lambda_{p}^{\prime}\right)\right\}, \quad I(\Lambda)=\left\{C \cdot N(x): x \in u\left(\Lambda_{p}\right)\right\} .
$$

We have seen that $C\left(\Lambda^{\prime}\right) \cong I(C, p) / I\left(\Lambda^{\prime}\right), C(\Lambda) \cong I(C, p) / I(\Lambda)$, and that $D(\Lambda) \cong$ $I\left(\Lambda^{\prime}\right) / I(\Lambda)$. Now

$$
\Lambda_{p}^{\prime} \cong \sum_{i=1}^{m}\left(\Lambda_{i}\right)_{p}, \quad\left(\Lambda_{i}\right)_{p} \cong \text { full matrix algebra over }\left(R_{i}\right)_{p},
$$

from which it is clear that $I\left(\Lambda^{\prime}\right)$ consists of all principal $C$-ideals in $F$ which are prime to $p$. Therefore,

$$
C\left(\Lambda^{\prime}\right) \cong \prod_{i=1}^{m} C\left(R_{i}\right)
$$

where $C\left(R_{i}\right)$ is the ideal class group of $R_{i}$.

One of our main results is as follows:

(5.3) Theorem. Let $G$ be an odd p-group. Then $|D(Z G)|$ is a power of $p$ (possibly equal to 1 ). 
This theorem was established by Fröhlich [8] for the case where $G$ is an abelian $p$-group. We devote the remainder of this section to proving the result for an arbitrary $p$-group, $p$ odd. In the next section, we shall prove the corresponding result for the case where $p=2$.

(5.4) Lemma. There is an epimorphism of multiplicative groups

$$
\theta: 1+\operatorname{rad} \Lambda_{p}^{\prime} \rightarrow I\left(\Lambda^{\prime}\right) / I(\Lambda)
$$

given by

$$
\theta(x)=C \cdot N(x) \bmod I(\Lambda), \quad x \in 1+\operatorname{rad} \Lambda_{p}^{\prime} .
$$

Proof. First of all, it is clear that $1+\operatorname{rad} \Lambda_{p}^{\prime}$ is a multiplicative group. Next, each $x \in 1+\operatorname{rad} \Lambda^{\prime}{ }_{p}$ is a unit in $\Lambda^{\prime}{ }_{p}$, and hence $C \cdot N(x) \in I\left(\Lambda^{\prime}\right)$. Therefore, $\theta$ maps $1+\operatorname{rad} \Lambda^{\prime}{ }_{p}$ into $I\left(\Lambda^{\prime}\right) / I(\Lambda)$. Since the reduced norm map $N$ is multiplicative, $\theta$ is a homomorphism, and we need to prove that $\theta$ is an epimorphism.

Let $C a \in I\left(\Lambda^{\prime}\right)$, where $\alpha \in F$ is prime to $p$. Then $\alpha=\Sigma \alpha_{i}$, with $\alpha_{i} \in K_{i}$ prime to $P_{i}$ (using the notation of (5.2)). Fix the notation so that $A_{1}=K_{1}=Q$, and $K_{i} \neq Q$ for $i>1$. Choose $r \in Z$ prime to $p$ so that $r a \in C$ and $r a_{1} \equiv 1(\bmod p)$. If we replace $C \alpha$ by $C \cdot \alpha N(r)$, the coset $\bmod I(\Lambda)$ is unchanged, but now each new $\alpha_{i}$ is an element of $R_{i}$, and $\alpha_{1} \equiv 1(\bmod p)$.

Changing notation, consider the element $C \alpha \in I\left(\Lambda^{\prime}\right)$, where $\alpha=\Sigma a_{i}$, with each $\alpha_{i} \in R_{i}$ prime to $P_{i}$, and where $\alpha_{1} \equiv 1(\bmod p)$. By $(5.2)$ we can choose $\beta_{i} \in R_{i}$ with

$$
\beta_{i} \equiv 1\left(\bmod P_{i}\right), \quad R_{i} \cdot \beta_{i}=R_{i} \cdot \alpha_{i} \text { for all } i .
$$

(For $i=1$, take $\beta_{1}=\alpha_{1}$.) Letting $\beta=\Sigma \beta_{i}$, we see that $C \alpha=C \beta$, and hence it suffices to show the existence of an element $x \in 1+\operatorname{rad} \Lambda^{\prime}{ }_{p}$ such that $N(x)=\beta$.

We may write

$$
\Lambda^{\prime}=\sum^{\cdot} \Lambda_{i}, \quad\left(\Lambda^{\prime}\right)_{p}=\sum^{\cdot}\left(\Lambda_{i}\right)_{p}=\sum\left(\Lambda_{i}\right)_{P_{i}}
$$

and thus

$$
\operatorname{rad} \Lambda_{p}^{\prime}=\sum \operatorname{rad}\left(\Lambda_{i}\right)_{P_{i}}
$$

Now each $A_{i}$ is a full matrix algebra over $K_{i}$, and so the theory of maximal orders tells us that each $\left(\Lambda_{i}\right)_{P_{i}}$ is a full matrix ring over $\left(R_{i}\right)_{P_{i}}$, and furthermore $\operatorname{rad}\left(\Lambda_{i}\right)_{P_{i}}=P_{i} \cdot\left(\Lambda_{i}\right)_{P_{i}} \cdot$. For each $i$, choose $x_{i} \in 1+P_{i} \cdot\left(\Lambda_{i}\right)_{P_{i}} \subset A_{i}$ such that $x_{i}$ is represented by a diagonal matrix with diagonal entries $\beta_{i}, 1, \ldots, 1$. Then $N_{i}\left(x_{i}\right)=\beta_{i}$, and setting $x=\Sigma x_{i}$, we have found an element $x \in 1+\operatorname{rad} \Lambda^{\prime}{ }_{p}$ such that $N(x)=\beta$. This completes the proof of the lemma.

(5.5) Lemma. Let $\Gamma$ be a ring with unity, and let $L$ be a left ideal of $\Gamma$ such that $L \subset \operatorname{rad} \Gamma$. Define $1+L=\{1+x: x \in L\}$. Then $1+L$ is a multiplicative group contained in $\Gamma$. 
Proof. Clearly $1+L$ is closed under multiplication, and we need only check the existence of inverses. Each $x \in L$ lies in $\operatorname{rad} \Gamma$, so $1+x$ has a two-sided inverse $w$ in $\Gamma$. Then $w(1+x)=1$, so $w=1-w x \in 1+L$, as desired.

(5.6) Lemma. Let us set $X^{\prime}=\operatorname{rad} \Lambda_{p}^{\prime}, X=X^{\prime} \cap \Lambda_{p}$. Then $1+X$ is a subgroup of the multiplicative group $1+X^{\prime}$, of index a finite power of $p$.

Proof. We imitate Fröhlich's proof in [8]. For sufficiently large $t$, we have

$$
\left(X^{\prime}\right)^{t} \subset p \cdot \Lambda_{p}^{\prime}, \quad p^{t} \cdot \Lambda_{p}^{\prime} \subset \Lambda_{p}
$$

Thus for large $r$ we obtain

$$
\left(X^{\prime}\right)^{r} \subset p \cdot \Lambda_{p}, \quad X^{r} \subset p \cdot \Lambda_{p} \subset \operatorname{rad} \Lambda_{p}
$$

Since $X$ is a two-sided ideal of $\Lambda_{p}$, and $X^{r} \subset \operatorname{rad} \Lambda_{p}$, it follows that also $X \subset$ $\operatorname{rad} \Lambda_{p}$. Hence $1+X$ is a multiplicative group, by (5.5).

For $i \geq 1$, we observe that $\left(X^{\prime}\right)^{i}+X$ is a two-sided ideal of the ring $\left(X^{\prime}\right)^{i}+$ $\Lambda_{p}$, and

$$
\left\{\left(X^{\prime}\right)^{i}+X\right\}^{r+i} \subset\left(X^{\prime}\right)^{r+i}+X^{r+i} \subset p\left\{\left(X^{\prime}\right)^{i}+\Lambda_{p}\right\} \subset \operatorname{rad}\left\{\left(X^{\prime}\right)^{i}+\Lambda_{p}\right\} .
$$

It follows from (5.5) that $1+\left(X^{\prime}\right)^{i}+X$ is a multiplicative subgroup of $1+X^{\prime}$.

Now consider the epimorphism

$$
\mu: 1+\left(X^{\prime}\right)^{i}+X \rightarrow\left(\left(X^{\prime}\right)^{i}+X\right) /\left(\left(X^{\prime}\right)^{i+1}+X\right)
$$

defined by

$$
\mu(1+x)=x+\left(X^{\prime}\right)^{i+1}+X, \quad x \in\left(X^{\prime}\right)^{i}+X .
$$

It is easily seen that $\mu$ is a homomorphism of a multiplicative group onto an additive group, and that

$$
\text { kernel of } \mu=1+\left(X^{\prime}\right)^{i+1}+X \text {. }
$$

Therefore

$$
\frac{1+\left(X^{\prime}\right)^{i}+X}{1+\left(X^{\prime}\right)^{i+1}+X} \cong \frac{\left(X^{\prime}\right)^{i}+X}{\left(X^{\prime}\right)^{i+1}+X} .
$$

Since $\left(X^{\prime}\right)^{r} \subset X$ by (5.7), we have

$$
\begin{aligned}
{\left[1+X^{\prime}: 1+X\right] } & =\prod_{i=1}^{r-1}\left[1+\left(X^{\prime}\right)^{i}+X: 1+\left(X^{\prime}\right)^{i+1}+X\right] \\
& =\prod_{i=1}^{r-1}\left[\left(X^{\prime}\right)^{i}+X:\left(X^{\prime}\right)^{i+1}+X\right]
\end{aligned}
$$


with the last equality a consequence of (5.8). Thus

$$
\left[1+X^{\prime}: 1+X\right]=\left[X^{\prime}: X\right] \text {. }
$$

But $p^{t} X^{\prime} \subset X$, and $X^{\prime}$ is finitely generated over $R$. Hence the index $\left[X^{\prime}: X\right]$ is a finite power of $p$, and the lemma is established.

We are now ready to conclude the proof of our Theorem 5.3. We have just shown that $1+X$ is a subgroup of $1+X^{\prime}$, of $p$-power index. Denote by $(1+X)^{*}$ the normal closure of $1+X$ in $1+X^{\prime}$. Then $(1+X)^{*}$ is generated by elements of the form $y(1+x) y^{-1}, y \in 1+X^{\prime}, x \in X$. Let $\theta: 1+X^{\prime} \rightarrow I\left(\Lambda^{\prime}\right) / I(\Lambda)$ be the epimorphism defined in (5.4). Then

$$
\begin{aligned}
\theta\left\{y(1+x) y^{-1}\right\} & =C \cdot N(1+x) \bmod I(\Lambda), \\
& =1 \bmod I(\Lambda),
\end{aligned}
$$

since $1+x \in u\left(\Lambda_{p}\right)$. Hence $\theta$ induces an epimorphism

$$
\left(1+X^{\prime}\right) /(1+X)^{*} \rightarrow I\left(\Lambda^{\prime}\right) / I(\Lambda) \cong D(\Lambda) .
$$

Since $\left[1+X^{\prime}:(1+X)^{*}\right]$ is a divisor of $\left[1+X^{\prime}: 1+X\right]$, it follows from (5.6) that $\left(1+X^{\prime}\right) /(1+X)^{*}$ is a finite $p$-group. Hence $D(\Lambda)$ is also a finite $p$-group, and the theorem is proved.

Professor L. McCulloh has suggested an alternative proof of (5.3). Denote by $I_{0}(C, F)$ the subgroup consisting of all principal ideals of $I(C, F)$, and by $S(C, F)$ the subgroup of $I_{0}(C, F)$ consisting of ideals which possess a generator $\alpha$ such that $\alpha \equiv$ 1 (mod*F).(4) Those ideals $C \cdot \Sigma \alpha_{i}, a_{i} \in K_{i}$, which satisfy the additional condition that $\left(\alpha_{i}\right)_{\hat{P}}>0$ at those infinite primes $P$ of $K_{i}$ ramified in $A_{i}$, form a subgroup denoted by $S^{+}(C, F)$. Of course, $I_{0}, S, S^{+}$are the direct sums of the corresponding groups for each simple component.

In [9, proof of Lemma 2.6] Jacobinski proved that $I(\Lambda) \supset S^{+}(C, F)$ if $A$ satisfies the Eichler condition, so we have a chain of ideal groups

$$
I_{0}(C, F) \supset I\left(\Lambda^{\prime}\right) \supset I(\Lambda) \supset S^{+}(C, F) \text {. }
$$

Now take $\Lambda=Z G, G$ an odd $p$-group of order $p^{n}$. In this case $s(C, F)=S^{+}(C, F)$. We first show that $I_{0}\left(R_{i}, F\right) / S\left(R_{i}, F\right)$ is a (finite) p-group for $i>1$. By (7.12) we must show that $u\left(R_{i} / p^{n} R_{i}\right) / u^{\prime}\left(R_{i}\right)$ is a $p$-group. Now the numerator has order equal to $(p-1)$ times a $p$-power. Since $u^{\prime}\left(R_{i}\right)$ contains the subgroup of order $p-1$ generated by the cyclotomic units $\left(1-\omega^{r}\right) /(1-\omega), r$ prime to $p$, it follows that $u\left(R_{i} / p^{n} R_{i}\right) / u^{\prime}\left(R_{i}\right)$ is a $p$-group. (We have used (5.1) and (5.2).)

Now $A_{1}=K_{1}=Q$ and

$$
I_{0}\left(Z, p^{n} Z\right) / S\left(Z, p^{n} Z\right) \cong u\left(Z / p^{n} Z\right) /\{ \pm 1\} .
$$

(4) This notation is explained in $\S 7$ just before (7.12). 
The second paragraph of the proof of (5.4) shows that the $A_{1}$-component of any element of $I\left(\Lambda^{\prime}\right)$ modulo $I(\Lambda)$ has a generator which is integral and $\equiv 1(\bmod p)$. It follows that $I\left(\Lambda^{\prime}\right) / I(\Lambda)$ is a $p$-group, as claimed.

6. The case $p=2$. We now turn to the more difficult case where $G$ is a finite 2-group, again setting $A=Q G, \Lambda=Z G$, and keeping the notation of $\$ 3$. For certain choices of $G$, the algebra $A$ may fail to satisfy the Eichler condition, and thus we must use Theorem 3.11 rather than (3.7) in order to calculate $D(\Lambda)$. We have $D(\Lambda) \cong J\left(\Lambda^{\prime}\right) / J(\Lambda)$, where

$$
\begin{aligned}
J\left(\Lambda^{\prime}\right) & =\left\{C \cdot N^{*}(x): x=\text { unit in } E\left(\Lambda^{\prime}{ }_{2}+\Lambda^{\prime}{ }_{2}\right)\right\}, \\
J(\Lambda) & =\left\{C \cdot N^{*}(x): x=\text { unit in } E\left(\Lambda_{2}+\Lambda_{2}\right)\right\} .
\end{aligned}
$$

We are going to prove the analogue of Theorem 5.3 for the present case, namely:

(6.1) Theorem. For any 2-group $G$, the order of $D(Z G)$ is a power of 2 (possibly equal to 1).

To start with, we need a pair of preliminary results.

(6.2) Theorem. Let $G$ be a 2-group. For each $i$, the center $K_{i}$ of the ith simple component $A_{i}$ of $A$ is a subfield of a cyclotomic field $Q(\omega)$, where $\omega$ is some $2^{n}$ th root of unity. There is a unique prime ideal $P_{i}$ of $R_{i}$ containing 2, and $R_{i} / P_{i} \cong Z / 2 Z$. Each $a \in R_{i}$ which is prime to $P_{i}$ satisfies the congruence $\alpha \equiv 1\left(\bmod P_{i}\right)$.

Proof. The field $K_{i}$ is obtained from $Q$ by adjoining values of irreducible complex characters of $G$ (see [3] or [6]). Therefore, $K_{i} \subset Q(\omega)$ for some $2^{n}$ th root of unity $\omega$. The remaining assertions are obvious, since 2 ramifies completely in $Q(\omega)$, hence also in $K_{i}$.

(6.3) Theorem (Eichler [5]). Let $S=\operatorname{alg}$ int $\{L\}$, and let $\Gamma$ be a maximal $S$ order in the simple algebra $B$ with center $L$. Assume that $B$ satisfies the Eichler condition. Let $N: B \rightarrow L$ be the reduced norm map, and let a be any two-sided ideal of $\Gamma$. Let $x \in \Gamma$ be such that

$$
N(x) \equiv \text { unit of } S(\bmod S \cap a) \text {. }
$$

Then there exists a unit $u$ of $\Gamma$ such that $x \equiv u(\bmod a)$.

Taking this result for granted, we continue with the proof of Theorem 6.1. We have seen in $\$ 3$ that $E(A+A)$ is a semisimple algebra with center $F$, and that $A$ satisfies the Eichler condition. Furthermore, $E\left(\Lambda^{\prime}+\Lambda^{\prime}\right)$ is a maximal $R$-order in $E(A+A)$. To simplify the notation, let us write

$$
E^{\prime}=E\left(\Lambda^{\prime}+\Lambda^{\prime}\right)=\sum_{i=1}^{m} E^{i}, \text { where } E^{i}=E\left(\Lambda_{i}+\Lambda_{i}\right) \text {. }
$$


Let $N_{i}{ }^{*}: E\left(A_{i}+A_{i}\right) \rightarrow K_{i}$ be the reduced norm map, and define $N^{*}: E(A+A) \rightarrow F$ by setting $N^{*}=\Sigma^{*} N_{i}^{*}$.

(6.4) Lemma. There is an epimorphism of multiplicative groups

$$
\varphi: 1+\operatorname{rad} E^{\prime}{ }_{2} \rightarrow J\left(\Lambda^{\prime}\right),
$$

given by $\varphi(y)=C \cdot N^{*}(y), y \in 1+\operatorname{rad} E^{\prime}{ }_{2}$. Here, $E^{\prime}{ }_{2}$ denotes the localization of $E^{\prime}$ at the rational prime 2.

Proof. As in the proof of (5.4), it is clear that $\varphi$ gives a multiplicative homomorphism of the group $1+\operatorname{rad} E^{\prime}{ }_{2}$ into the group $J\left(\Lambda^{\prime}\right)$. This latter group is generated by the set of elements

$$
\left\{C \cdot N^{*}(x): x \in E^{\prime}, x \text { prime to } 2\right\} \text {. }
$$

Hence, to show that $\varphi$ is epic, it suffices to show that for each such $x$ we can find an element $y \in 1+\operatorname{rad} E^{\prime}{ }_{2}$ such that $C \cdot N^{*}(y)=C \cdot N^{*}(x)$.

We observe that

$$
E_{2}^{\prime}=\sum^{\cdot}\left(E^{i}\right)_{2}=\sum^{\cdot}\left(E^{i}\right)_{P_{i}}, \quad \operatorname{rad} E_{2}^{\prime}=\sum \operatorname{rad}\left(E^{i}\right)_{P_{i}},
$$

with the $\left\{P_{i}\right\}$ defined as in (6.2). The entire computation can be performed componentwise, so our problem reduces to the following:

Given $x_{i} \in E^{i}$ prime to $P_{i}$, show that there exists an element $y_{i} \in 1+$ $\operatorname{rad}\left(E^{i}\right)_{P_{i}}$ such that

$$
R_{i} \cdot N_{i}^{*}\left(y_{i}\right)=R_{i} \cdot N_{i}^{*}\left(x_{i}\right)
$$

Now $N_{i}^{*}\left(x_{i}\right) \in R_{i}$ is prime to $P_{i}$ (since $x_{i}$ is prime to $P_{i}$ ), and therefore (by (6.2)) $N_{i}{ }^{*}\left(x_{i}\right) \equiv 1\left(\bmod P_{i}\right)$. We shall now use (6.3) for the case when $B=$ $E\left(A_{i}+A_{i}\right), \Gamma=E^{i}=E\left(\Lambda_{i}+\Lambda_{i}\right), L=K_{i}, S=R_{i}, a=P_{i} E^{i}, a \cap S=P_{i}$. It follows that there exists a unit $u_{i} \in E^{i}$ such that

$$
x_{i} \equiv u_{i}\left(\bmod P_{i} E^{i}\right)
$$

Hence $u_{i}^{-1} x_{i} \equiv 1\left(\bmod P_{i} E^{i}\right)$, and thus

$$
u_{i}^{-1} x_{i}-1 \in P_{i} E^{i} \subset \operatorname{rad}\left(E^{i}\right)_{P_{i}}
$$

Let us now set $y_{i}=u_{i}^{-1} x_{i}$, so $y_{i} \in 1+\operatorname{rad}\left(E^{i}\right)_{P_{i}}$. Since $N_{i}^{*}\left(u_{i}\right)$ is a unit in $R_{i}$, it is clear that (6.5) is valid. Thus $y_{i}$ has the desired properties, and if we set $y=\Sigma y_{i}$, then

$$
y \in 1+\operatorname{rad} E_{2}^{\prime}, \quad C \cdot N^{*}(y)=C \cdot N^{*}(x) .
$$

This completes the proof of the lemma. 
Remark. If we had used Eichler's Theorem 6.3 for the case where $p$ is odd, we would not have needed to use the result from (5.1) that $A_{i}$ is a full matrix algebra over $K_{i}$.

It is now an easy matter to complete the proof that $|D(Z G)|$ is a power of 2 . The argument given at the end of $\$ 5$ carries over unchanged to the present case, and shows that $\varphi$ induces an epimorphism

$$
\left(1+Y^{\prime}\right) /(1+Y)^{*} \rightarrow J\left(\Lambda^{\prime}\right) / J(\Lambda) \cong D(\Lambda)
$$

where

$$
Y^{\prime}=\operatorname{rad} E_{2}^{\prime}, \quad Y=Y^{\prime} \cap\{E(\Lambda+\Lambda)\}_{2},
$$

and $(1+Y)^{*}=$ normal closure of $1+Y$ in $1+Y^{\prime}$. As in $\$ 5$, the index $\left[1+Y^{\prime}\right.$ : $1+Y]$ is a power of 2 . Thus the order of $D(Z G)$ is also a power of 2 , as claimed.

Next we shall sketch an alternate proof of $(6.1)$, which parallels that given at the end of $\$ 5$. Set $E^{\prime}=E\left(\Lambda^{\prime}+\Lambda^{\prime}\right)$ and $E=E(\Lambda+\Lambda)$. The centers of $\Lambda^{\prime}$ and $E^{\prime}$ are isomorphic to $C$, and we have $J\left(\Lambda^{\prime}\right) \cong I\left(E^{\prime}\right), J(\Lambda) \cong I(E)$. Since $K E^{\prime}$ satisfies the Eichler condition, we have a chain

$$
I_{0}(C, F) \supset J\left(\Lambda^{\prime}\right) \supset J(\Lambda) \supset S^{+}(C, F) .
$$

Let $|G|=2^{n}, \quad F=2^{n} Z$. Using (7.12) and the fact that $\left|u\left(R_{i} / 2^{n} R_{i}\right)\right|$ is a power of 2, we see that $I_{0}\left(R_{i}, 2^{n} R_{i}\right) / S\left(R_{i}, 2^{n} R_{i}\right)$ is a 2-group for all $i$. But $S(C, f) / S^{+}(C, f)$ is also a 2-group, whence so is $J\left(\Lambda^{\prime}\right) / J(\Lambda)$, as desired.

7. Cyclic groups of order $2 p$. Throughout this section, let $p$ be an odd prime, and let $G$ be cyclic of order $2 p$. We shall calculate $C(Z G)$ and $D(Z G)$ explicitly, and we begin with a few elementary results on units in cyclotomic fields. To fix the notation, let $\omega$ be a primitive $p$ th root of unity, and set

$$
\begin{aligned}
& K=Q(\omega), \quad R=\operatorname{alg} \operatorname{int}\{K\}=Z[\omega], \quad P=(1-\omega) R, \\
& L=Q\left(\omega+\omega^{-1}\right), \quad S=\operatorname{alg} \operatorname{int}\{L\}=Z\left[\omega+\omega^{-1}\right] .
\end{aligned}
$$

Then $P$ is the unique prime ideal of $R$ containing $p$, and we have

$$
R / P \cong S /(P \cap S) \cong Z / p Z=\bar{Z} \quad \text { (say). }
$$

For each $r \in Z$, define

$$
\xi_{r}=\left(\omega^{r}-\omega^{-r}\right) /\left(\omega-\omega^{-1}\right) \in S .
$$

If $p \nmid_{r}$, then $\omega$ is a power of $\omega^{r}$, and hence $\xi_{r}^{-1} \in S$. Thus $\xi_{r}$ is a unit in $S$ whenever $p \nmid r$.

Furthermore, for each $r \in Z$ we obtain

$$
\xi_{r} \equiv r(\bmod P) .
$$


This follows at once from the equations

$$
\begin{aligned}
\xi_{r} & =\left(\omega^{-r} / \omega^{-1}\right) \cdot\left(1-\omega^{2 r}\right) /\left(1-\omega^{2}\right)=\omega^{1-r}\left(1+\omega^{2}+\omega^{4}+\cdots+\omega^{2 r-2}\right) \\
& \equiv r(\bmod P), \text { since } P=(1-\omega) R .
\end{aligned}
$$

Next, an elementary calculation yields

$$
\xi_{r}{ }^{2}-\xi_{s}{ }^{2}=\omega^{2-2 r} \cdot \frac{\omega^{2(r-s)}-1}{\omega^{2}-1} \cdot \frac{\omega^{2(r+s)}-1}{\omega^{2}-1} .
$$

If $r \equiv \pm s(\bmod p)$, this shows that $\xi_{r}{ }^{2}-\xi_{s}{ }^{2}=0$. If $r \not \equiv \pm s(\bmod p)$, each of the three factors on the right-hand side of the above displayed equations is a unit in $R$, and hence $\xi_{r}{ }^{2}-\xi_{S}{ }^{2}$ is a unit in $S$. We have thus proved the following result, which however will not be needed until $\$ 8$ :

(7.5) Lemma. For $r, s \in Z$, define $\xi_{r}, \xi_{s}$ as in (7.3). Then

$$
\xi_{r}^{2}-\xi_{s}^{2}= \begin{cases}0, & r \equiv \pm s(\bmod p), \\ \text { unit in } s, & \text { otherwise. }\end{cases}
$$

We may describe the cyclic group $G$ of order $2 p$ in terms of generators and relations; thus

$$
G=\left\langle\sigma, \tau: \sigma^{p}=1, \tau^{2}=1, \sigma \tau=\tau \sigma\right\rangle .
$$

Let $H=\langle\tau\rangle$ be the subgroup of $G$ of order 2. Our main result here is as follows:

(7.6) Theorem. Let $u^{\prime}(R)$ be the image of $u(R)$ in $u(R / 2 R)$. Then

$$
D(Z G) \cong u(R / 2 R) / u^{\prime}(R)
$$

and consequently

$$
|C(Z G)|=|C(R)|^{2}\left[u(R / 2 R): u^{\prime}(R)\right],
$$

where $C(R)$ is the ideal class group of $R$.

In proving Theorem 7.6, as well as in later calculations, we shall need some remarks on pullback diagrams (= fibre products). Let $a, b$ be two-sided ideals of an arbitrary ring $\Lambda$ with 1 , not necessarily commutative. Then it is easily verified that there is a pullback diagram

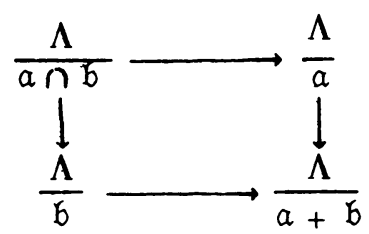

that is, 


$$
\Lambda /(a \cap \mathfrak{b}) \cong\{(x, y) \in(\Lambda / a)+(\Lambda / b): x \equiv y \bmod (a+b)\} .
$$

We apply this to the case where $\Lambda=Z G$, with $G$ cyclic of order $2 p$ as above. We pick

$$
a=\Phi(\sigma) \cdot \Lambda, \quad \mathfrak{b}=(\sigma-1) \cdot \Lambda,
$$

where $\Phi(X)=1+X+\cdots+X^{p-1}$ is the cyclotomic polynomial of order $p$. Since $Q G$ is free as $Q[\sigma]$-module, it is clear that $a \cap b=0$. Furthermore, there are ring is omorphisms

$$
\Lambda / a \cong R H, \quad \Lambda / b \cong Z H, \quad \Lambda /(a+b) \cong \bar{Z} H,
$$

with $\bar{Z}=Z / p Z \cong R / P$. The first of these isomorphisms is obtained by letting $\sigma \rightarrow \omega$, the second by $\sigma \rightarrow 1$, the third by $\sigma \rightarrow 1, Z \rightarrow \bar{Z}$. The pullback diagram (7.7) becomes

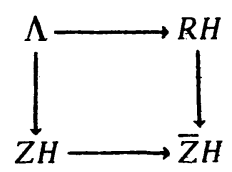

We shall use bars to indicate images of elements of $Z H$ or $R H$ in $\bar{Z} H$. Tensoring with $Q$, we see that $Q G$ can be identified with $Q H+K H$, and that the maximal order $\Lambda^{\prime}$ of $Q G$ may be identified with $Z^{(2)}+R^{(2)}$. Further,

$$
\Lambda \cong\{(\xi, \eta) \in Z H+R H: \bar{\xi}=\bar{\eta} \text { in } \bar{Z} H\}
$$

Once $\Lambda^{\prime}$ has been identified with $Z^{(2)}+R^{(2)}$, it remains for us to describe $\Lambda$ as a subring of $\Lambda^{\prime}$. Now $Q H \cong Q^{(2)}, K H \cong K^{(2)}$, and

$$
\begin{aligned}
& Z H \cong\left\{\left(a_{0}, a_{1}\right) \in Z^{(2)}: a_{0} \equiv a_{1}(\bmod 2 Z)\right\}, \\
& R H \cong\left\{\left(b_{0}, b_{1}\right) \in R^{(2)}: b_{0} \equiv b_{1}(\bmod 2 R)\right\} .
\end{aligned}
$$

It follows from these isomorphisms, together with (7.10), that we may identify $\Lambda$ with the subring of $\Lambda^{\prime}$ consisting of all 4-tuples $\left(a_{0}, a_{1}, b_{0}, b_{1}\right) \in Z^{(2)}+R^{(2)}$ which satisfy the congruence conditions

$$
a_{0} \equiv a_{1}(\bmod 2 Z), \quad b_{0} \equiv b_{1}(\bmod 2 R), \quad a_{0} \equiv b_{0}, a_{1} \equiv b_{1}(\bmod P) .
$$

Now $D(\Lambda) \cong I\left(\Lambda^{\prime}\right) / I(\Lambda)$ by Theorem 3.7. In our particular case, $C=\Lambda^{\prime}$ and the reduced norm map $N$ is the identity map. Thus

$$
I\left(\Lambda^{\prime}\right)=\left\{\Lambda^{\prime} \cdot x: x \in u\left(\Lambda_{2 p}^{\prime}\right)\right\}, \quad I(\Lambda)=\left\{\Lambda^{\prime} \cdot y: y \in u\left(\Lambda_{2 p}\right)\right\} .
$$

Denote by $I_{0}(R, C)$ the group of all principal $R$-ideals in $K$ prime to the ideal $c$ of $R$, and define $I_{0}(Z, m)$ analogously as the group of $Z$-ideals in $Q$ prime to the integer $m$. Since $\Lambda^{\prime}=Z^{(2)}+R^{(2)}$, we have

$$
I\left(\Lambda^{\prime}\right)=\left\{I_{0}(Z, 2 p)\right\}^{(2)}+\left\{I_{0}(R, 2 P)\right\}^{(2)} \text {. }
$$


Before proceeding further, we require two lemmas, the first of which is a general result true for any algebraic number field $\Omega$. Let $\Omega=\operatorname{alg} \operatorname{int}\{\Omega\}$, let $q$ be a nonzero ideal of $\supseteqq$, and write $q=\Pi p_{i}{ }^{e_{i}}, e_{i}>0$, with the $\left\{p_{i}\right\}$ distinct prime ideals of $\Omega$. Let $v_{i}$ be the normalized $p_{i}$-adic valuation on $\Omega$. For $x \in \Omega$, we shall write $x \equiv 1\left(\bmod ^{*} q\right)$, if and only if $v_{i}(x-1) \geq e_{i}$ for each $p_{i}$ dividing $q$. Let $I_{0}(\bigcap, q)$ be the group of principal $\supseteqq$-ideals in $\Omega$ prime to $q$, and let $S(\bigcap, q)$ be the subgroup of $I_{0}(\bigcap, q)$ defined by

$$
S(\bigcap, q)=\left\{x \supseteqq: x \in \Omega, x \neq 0, x \equiv 1 \bmod ^{*} q\right\} .
$$

(7.12) Lemma. Let $q$ and $x$ be relatively prime ideals of $\supseteqq$, and let $u^{\prime}(\Omega)$ be the image of $u(\supseteqq)$ in $u(\supseteqq / q)$. Then

$$
\frac{I_{0}(\Omega, q x)}{I_{0}(\Omega, q x) \cap S(\Omega, q)} \cong \frac{u(\Omega / q)}{u^{\prime}(\Omega)} .
$$

Hence $I_{0}(\bigcap, q x) \subset S(\supseteq, q)$ whenever $u^{\prime}(\supseteq)=u(\supseteq / q)$.

Proof. Define a mapping $\kappa: I_{0}(\bigcap, q x) \rightarrow u(\bigcap / q) / u^{\prime}(\bigcap)$ by setting $\kappa(x \supseteqq)=$ coset containing $\bar{x}$, where the element $x \in \Omega$ prime to $q x$ maps onto $\bar{x} \in u(\bigcap / q)$. Clearly $\kappa$ is a well-defined homomorphism. Further, $\kappa$ is epic, since given any $\alpha \in u(\Omega / q)$, we can find an element $x \in \Omega$ such that $\bar{x}=\alpha, x \equiv 1\left(\bmod ^{*} x\right)$, and then $\kappa(x \cong)$ equals the coset containing $\alpha$.

It remains to determine ker $\kappa$. Let $\kappa(x \Omega)=1$, where $x \in \Omega$ is prime to $q x$. Then $\bar{x} \in u^{\prime}(\supseteq)$; that is, $x \equiv u\left(\bmod ^{*} q\right)$ for some $u \in u(\supseteqq)$. But then $x \supseteqq=$ $x u^{-1} . \supseteqq \in S(\supseteqq, q)$, which proves that

$$
\operatorname{ker} \kappa \subset I_{0}(\bigcap, q x) \cap S(\bigcap, q) .
$$

The reverse inclusion is obvious, so the lemma is established.

Now observe that $u^{\prime}(R)=u(R / P)$ by $(7.4)$, so by the second statement in the lemma we obtain

$$
I_{0}(R, 2 P) \subset S(R, P) .
$$

We shall use this in a moment.

(7.14) Lemma. Let $u^{\prime \prime}(R)$ be the image in $u(R / 2 R)$ of the group $\{u \in u(R)$ : $u \equiv 1(\bmod P)\}$. Then $u^{\prime \prime}(R)=u^{\prime}(R)$.

Proof.(5) Obviously $u^{\prime \prime}(R) \subset u^{\prime}(R)$, and we must prove the reverse inclusion. Let $u \in u(R)$ be given; we need to find a unit $v$ in $R$ such that $v \equiv u(\bmod 2 R)$, $v \equiv 1(\bmod P)$. Let $\theta$ be the automorphism of $K$ defined by letting $\omega \rightarrow \omega^{2}$. Then

$$
u^{\theta} \equiv u^{2}(\bmod 2 R), \quad u^{\theta} \equiv u(\bmod P),
$$

(5) The authors wish to thank Professor L. McCulloh for providing this simple proof. 
and we need only choose $v=u^{\theta} / u$.

We shall now complete the proof of Theorem 7.6 by constructing an epimorphism

$$
\psi: I\left(\Lambda^{\prime}\right) \rightarrow u(R / 2 R) / u^{\prime}(R)
$$

with kernel $I(\Lambda)$. Let bars denote images in $u(R / 2 R)$, and define

$$
\psi\left(Z a_{0}, Z a_{1}, R b_{0}, R b_{1}\right)=\text { coset containing } \bar{b}_{0} / \bar{b}_{1},
$$

where $a_{i} \in u\left(Z_{2 p}\right), b_{i} \in u\left(R_{2 P}\right)$. Clearly, $\psi$ is a homomorphism. It is epic because the map $I_{0}(R, 2 P) \rightarrow u(R / 2 R) / u^{\prime}(R)$ is already epic, as follows from the proof of (7.12).

We see from (7.11) that $u\left(\Lambda_{2 p}\right)$ consists of all 4-tuples

$$
\left(a_{0}, a_{1}, b_{0}, b_{1}\right), \quad a_{i} \in u\left(Z_{2 p}\right), b_{i} \in u\left(R_{2 P}\right),
$$

which satisfy the conditions

$$
a_{0} \equiv a_{1}\left(\bmod ^{*} 2 Z\right), \quad b_{0} \equiv b_{1}\left(\bmod ^{*} 2 R\right), \quad a_{0} \equiv b_{0}, a_{1} \equiv b_{1}\left(\bmod ^{*} P\right) .
$$

For each such 4-tuple we have $\bar{b}_{0}=\bar{b}_{1}$, whence $I(\Lambda) \subset \operatorname{ker} \psi$. To prove the reverse inclusion, let $\left(Z a_{0}, Z a_{1}, R b_{0}, R b_{1}\right) \in I\left(\Lambda^{\prime}\right)$ lie in the kernel of $\psi$. By (7.13), there exist elements $c_{0}, c_{1} \in K$ such that

$$
R b_{i} a_{i}^{-1}=R c_{i}, \quad c_{i} \equiv 1\left(\bmod ^{*} P\right), \quad i=1,2 .
$$

Hence $R b_{i}=R a_{i} c_{i}$, so replacing $b_{i}$ by $a_{i} c_{i}$, we may henceforth assume that $a_{0} \equiv b_{0}, a_{1} \equiv b_{1}\left(\bmod ^{*} P\right)$. But $\psi\left(Z a_{0}, Z a_{1}, R b_{0}, R b_{1}\right)=1$ implies that $\bar{b}_{0} / \bar{b}_{1} \epsilon$ $u^{\prime}(R)$, so by (7.14) there exists a unit $u \in R$ such that $b_{0} \equiv b_{1} u\left(\bmod ^{*} 2 R\right), u \equiv$ $1(\bmod P)$. Replacing $b_{1}$ by $b_{1} u$ does not affect the congruence $a_{1} \equiv b_{1}\left(\bmod ^{*} P\right)$, and permits us to assume that $b_{0} \equiv b_{1}\left(\bmod ^{*} 2 R\right)$. Since trivially $a_{0} \equiv$ $a_{1}\left(\bmod ^{*} 2 Z\right)$, we may conclude that the 4-tuple $\left(a_{0}, a_{1}, b_{0}, b_{1}\right)$ satisfies all of the conditions in (7.15), and hence that $\left(Z a_{0}, Z a_{1}, R b_{0}, R b_{1}\right) \in I(\Lambda)$. This completes the proof of the assertion about $D(\Lambda)$. The second assertion in (7.6) is then obvious, since $C\left(\Lambda^{\prime}\right) \cong C(R) \times C(R)$.

As a matter of fact, the epimorphism $\Lambda \rightarrow R H$ induces epimorphisms $D(Z G)$ $\rightarrow D(R H), C(Z G) \rightarrow C(R H)$. Ullom [16] has previously shown that

$$
|D(R H)|=\left[u(R / 2 R): u^{\prime}(R)\right],
$$

from which we may conclude that $D(Z G) \cong D(R H)$ and $C(Z G) \cong C(R H)$. These latter conclusions can also be obtained directly from (7.9) by use of Milnor's Mayer-Vietoris sequence (see [18]), but one is then left with the problem of calculating $D(R H)$.

8. Dihedral groups of order $2 p$. Let $G$ be the dihedral group of order $2 p$, where $p$ is an odd prime. We shall again use the notation introduced in (7.1), so 
that $S$ is the ring of algebraic integers in the subfield $L$ of the cyclotomic field $K$. The class group $C(S)$ is then the ideal class group of $S$. We shall prove

$$
C(Z G) \cong C(S), \quad D(Z G)=1 \text {. }
$$

These results are immediate consequences of Lee's classification [10] of ZGlattices. Our proof is more direct, but of course provides no information about the nonprojective $Z G$-lattices.

Throughout this section, we put

$$
G=\left\langle\sigma, \tau: \sigma^{p}=1, \tau^{2}=1, \tau \sigma \tau^{-1}=\sigma^{-1}\right\rangle, \quad H=\left\langle\tau: \tau^{2}=1\right\rangle,
$$

and set $A=Q G, \Lambda=Z G$. Let $x \rightarrow x^{\prime}$ be the $L$-automorphism of $K$ induced by the map $\omega \rightarrow \omega^{-1}$ (see (7.1)). We introduce the twisted group algebra

$$
K\langle\tau\rangle=K \oplus K \tau, \quad \tau x=x^{\prime} \cdot \tau, \quad x \in K .
$$

Then $K\langle\tau\rangle$ is a simple algebra with center $L$, and is split by $K$ :

$$
K\langle\tau\rangle \cong \operatorname{Hom}_{L}(K, K)
$$

The isomorphism is obtained by mapping $x+y \tau \in K\langle\tau\rangle$ onto $\phi_{x+y \tau}$, where $\phi_{x+y \tau}(u)=x u+y u^{\prime}, u \in K$. On the other hand, $K\langle\tau\rangle$ is a cyclic algebra, and as such has a matrix representation over $K$ given by

$$
x+y r \rightarrow\left(\begin{array}{cc}
x & y \\
y^{\prime} & x^{\prime}
\end{array}\right), \quad x, y \in K .
$$

If $N: K\langle\tau\rangle \rightarrow L$ is the reduced norm map, the above implies that

$$
N(x+y \tau)=\left|\begin{array}{cc}
x & y \\
y^{\prime} & x^{\prime}
\end{array}\right|=x x^{\prime}-y y^{\prime} .
$$

We may make $K\langle\tau\rangle$ into a $Q G$-module by letting $\sigma$ act as multiplication by $\omega$ on the coefficients from $K$. The decomposition of $A$ into simple components is then given by $A=Q+Q+K\langle\tau\rangle$. The first summand affords the trivial representation $\sigma \rightarrow 1, \tau \rightarrow 1$, while the second gives $\sigma \rightarrow 1, \tau \rightarrow-1$.

Let us use a pullback diagram to describe the elements of $\Lambda$ in terms of their projections into the simple components of $A$. Since the cyclic subgroup $[\sigma]$ generated by $\sigma$ is normal in $G$, the ideals $a, b$ of $\Lambda$ defined by (7.8) are twosided ideals of $\Lambda$. Furthermore, $a-b=0$ since $Q G$ is $Q[\sigma]$-free. Let

$$
R\langle\tau\rangle=R \oplus R \tau \subset K\langle\tau\rangle
$$

Then there are ring isomorphisms

$$
\Lambda / \mathfrak{b} \cong Z H, \quad \Lambda / a \cong R\langle\tau\rangle, \quad \Lambda /(a+b) \cong \bar{Z} H,
$$

with $\bar{Z}$ as in (7.2). The pullback diagram (7.7) then becomes 


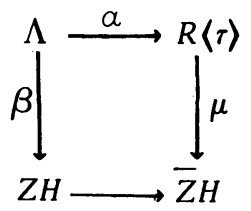

The map $\mu$ is given by $\mu(x+y r)=\bar{x}+\bar{y} r$, where $x \in R$ maps onto $\bar{x} \in R / P \cong \bar{Z}$. Since $x \equiv x^{\prime}(\bmod P)$ for $x \in R$, we see that $(\bmod P) \tau$ commutes with the elements of $R$, and hence $\bar{Z} H$ is an ordinary untwisted group algebra. Further, $\alpha$ is just the projection of $\Lambda$ into the simple component $K\langle\tau\rangle$, while $\beta$ is the projection into $Q+Q$, once we identify $Q H$ with $Q+Q$.

In the isomorphism $Q H \cong Q+Q$, the ring $Z H$ is identified with

$$
\left\{\left(a_{0}, a_{1}\right) \in Z+Z: a_{0} \equiv a_{1}(\bmod 2)\right\} .
$$

It then follows from (8.3) that we may identify $\Lambda$ with the ring of all triples $\left\{\left(a_{0}, a_{1}, x+y \tau\right) \in Z+Z+R\langle\tau\rangle\right\}$ satisfying the congruences

(8.4) $a_{0} \equiv a_{1}(\bmod 2), x \equiv\left(a_{0}+a_{1}\right) / 2(\bmod P), y \equiv\left(a_{0}-a_{1}\right) / 2(\bmod P)$.

Now let $\Lambda^{\prime}$ be a maximal $Z$-order in $A$ containing $\Lambda$. Since each simple component of $A$ is a full matrix algebra over its center, surely $A$ satisfies the Eichler condition. Furthermore, the discussion in $\$ 3(i)$ shows that

$$
\begin{aligned}
I\left(\Lambda^{\prime}\right) & =\{C \cdot \alpha: \alpha \in F, \alpha \text { prime to } 2 p\} \\
& =\left\{\left(Z b_{0}, Z b_{1}, S \beta\right): b_{0}, b_{1} \in Q, \beta \in L, \text { with } b_{0}, b_{1}, \beta \text { prime to } 2 p\right\} .
\end{aligned}
$$

On the other hand, $I(C, 2 p)$ consists of all $C$-ideals in $F$ which are prime to $2 p$. Therefore (by (3.7))

$$
C\left(\Lambda^{\prime}\right) \cong I(C, 2 p) / I\left(\Lambda^{\prime}\right) \cong C(Z) \times C(Z) \times C(S) \cong C(S)
$$

where $C(Z)=$ ideal class group of $Z$, and so on. By (3.7) we have $D(\Lambda) \cong$ $\dot{I}\left(\Lambda^{\prime}\right) / I(\Lambda)$, where

$$
I(\Lambda)=\{C \cdot N(x): x \in \Lambda, x \text { prime to } 2 p\} .
$$

We shall show below that $I(\Lambda)=I\left(\Lambda^{\prime}\right)$. This will imply that $D(\Lambda)=1$ and $C(\Lambda)$ $\cong C\left(\Lambda^{\prime}\right) \cong C(S)$, as claimed in (8.1).

(8.5) Lemma. (i) The map $\mu: R\langle\tau\rangle \rightarrow \bar{Z} H$ induces an epimorphism of unit groups: $u(R\langle\tau\rangle) \rightarrow u(\bar{Z} H)$.

(ii) Let $\beta \in S$ be prime to $p$. Then there exists an element $x+y \tau \in R\langle\tau\rangle$ such that $S \cdot N(x+y r)=S \beta$.

Proof. (i) Each element of $\bar{Z} H$ is of the form $\bar{r}+\bar{s} \tau$, where $r, s \in Z$ have images $\bar{r}, \bar{s} \in \bar{Z}$. Since $(\bar{r}+\bar{s} \tau)(\bar{r}-\bar{s} \tau)=\bar{r}^{2}-\bar{s}^{2}$, we see that $\bar{r}+\bar{s} \tau \in u(\bar{Z} H)$ if and only if $\bar{r}^{2} \neq \bar{s}^{2}$. Given such a unit $\bar{r}+\bar{s} \tau$, we may form $\xi_{r}+\xi_{s} \tau \in R\langle\tau\rangle$. Now $\tau$ commutes with the $\xi^{\prime}$ 's, since they lie in $S$. It follows from (7.5) that $\xi_{r}+\xi_{s} \tau$ is 
a unit in $R\langle\tau\rangle$, and clearly $\mu\left(\xi_{r}+\xi_{s} \tau\right)=\bar{r}+\bar{s} \tau$, as desired.

(ii) We have $S /(P \cap S) \cong \bar{Z}$, so $\beta \equiv r(\bmod P)$ for some $r \in Z$ with $p \nmid r$. The element $\xi_{r}$ given in $(7.3)$ is a unit in $S$ such that $\xi_{r} \equiv r(\bmod P)$. Setting $\alpha=$ $\beta \cdot \xi_{r}^{-1}$, we obtain

$$
S \cdot \beta=S \cdot \alpha, \quad \alpha \equiv 1(\bmod P) .
$$

It then suffices to find an element $x+y \tau \in R\langle\tau\rangle$ such that $N(x+y \tau)=\alpha$. We may set

$$
\alpha=1-\gamma\left(\omega-\omega^{-1}\right)^{2} /\left(\omega^{2}+\omega^{-2}\right)
$$

for some $\gamma \in S$. Then define $x=x_{0}+x_{1} \omega, y=y_{0}+y_{1} \omega$, where $x_{0}=1-\gamma, y_{0}=$ $a-x_{0}, x_{1}=-y_{1}=\left(\omega+\omega^{-1}\right) y_{0} / 2$. It is then easily checked that $x+y \tau \epsilon$ $R\langle\tau\rangle$, and $N(x+y \tau)=x x^{\prime}-y y^{\prime}=\alpha$.

Let $\lambda \in \Lambda$ correspond to the triple $\left(a_{0}, a_{1}, x+y \tau\right)$; then

$$
C \cdot N(\lambda)=Z a_{0}+Z a_{1}+S \cdot N(x+y \tau) \text {. }
$$

Further, $\lambda$ is prime to $2 p$ if and only if $a_{0}, a_{1}$, and $x+y r$ are prime to $2 p$. Now $I\left(\Lambda^{\prime}\right)$ is generated by ideals of the form

$$
Z b_{0}+Z b_{1}+S \beta, \quad b_{0}, b_{1}, \beta \text { prime to } 2 p \text {. }
$$

In order to show that $I\left(\Lambda^{\prime}\right)=I(\Lambda)$, we must show that given the ideal in (8.6), we can choose $a_{0}, a_{1}, x+y \tau$ prime to $2 p$, and satisfying (8.4), such that

$$
Z a_{0}=Z b_{0}, \quad Z a_{1}=Z b_{1}, \quad S \cdot N(x+y \tau)=S \beta .
$$

Indeed, we pick $a_{0}=b_{0}, a_{1}=b_{1}$, and we must find $x+y \tau \in R\langle\tau\rangle$ such that

$$
x \equiv\left(b_{0}+b_{1}\right) / 2, \quad y \equiv\left(b_{0}-b_{1}\right) / 2(\bmod P), \quad S \cdot N(x+y \tau)=S \cdot \beta .
$$

Let $c_{1}=\left(b_{0}+b_{1}\right) / 2, c_{2}=\left(b_{0}-b_{1}\right) / 2$, so $c_{1}{ }^{2}-c_{2}{ }^{2}=b_{0} b_{1}$, whence in $Z$ we have $\bar{c}_{1}{ }^{2}-\bar{c}_{2}{ }^{2} \neq 0$. Thus $\bar{c}_{1}+\bar{c}_{2} \tau \in u(\bar{Z} H)$. By (8.5)(ii) we may choose $x_{0}+$ $y_{0} \tau \in R\langle\tau\rangle$ such that $S \cdot N\left(x_{0}+y_{0} \tau\right)=S \cdot \beta$. Hence $x_{0} x_{0}^{\prime}-y_{0} y_{0}^{\prime}$ is prime to $p$, whence $\bar{x}_{0}+\bar{y}_{0} \tau \in u(\bar{Z} H)$. By $(8.5)(\mathrm{i})$, we may choose $u \in u(R\langle\tau\rangle)$ such that $\left(\bar{x}_{0}+\bar{y}_{0} \tau\right) \cdot \mu(u)=\bar{c}_{1}+\bar{c}_{2} \tau$. Set $x+y \tau=\left(x_{0}+y_{0} \tau\right) u$; since $N(u) \in u(S)$, this gives $S \cdot N(x+y \tau)=S \cdot N\left(x_{0}+y_{0} \tau\right)=S \cdot \beta$, and furthermore, $\bar{x}+\bar{y} \tau=\bar{c}_{1}+\bar{c}_{2} \tau$, that is, $x \equiv c_{1}(\bmod P), y \equiv c_{2}(\bmod P)$, as desired. This completes the proof of (8.1).

9. The quaternion group of order 8 . Our main result here is as follows:

(9.1) Theorem. Let $G$ be the quaternion group of order 8. Then $C(Z G)$ bas order 2 , and equals $D(Z G)$.

We may write

$$
G=\left\langle\sigma, \tau: \sigma^{4}=1, \sigma^{2}=\tau^{2}, \tau \sigma \tau^{-1}=\sigma^{-1}\right\rangle
$$


The group algebra $A=Q G$ splits into a direct sum of five simple components:

$$
A \cong Q^{(4)}+S, \quad S=Q \oplus Q i \oplus Q j \oplus Q k .
$$

The first four components correspond to the four distinct one-dimensional representations of $G$, namely $\sigma \rightarrow \pm 1, \tau \rightarrow \pm 1$. The fifth component $S$ is the skewfield of rational quaternions, corresponding to the representation $\sigma \rightarrow i, \tau \rightarrow j$.

It is more convenient for us to use the isomorphism

$$
f: Q G \cong Q V+S,
$$

where $V=\left\langle s, t: s^{2}=t^{2}=1\right.$, st $\left.=t s\right\rangle$ is an abelian $(2,2)$-group, and where the isomorphism $f$ is given by $\sigma \rightarrow(s, i), \tau \rightarrow(t, j)$. We will henceforth identify $Q G$ with $Q V+S$, and view ordered pairs $(\nu, \alpha) \in Q V+S$ as elements of $Q G$.

Now let $e_{0}, e_{1}, e_{2}, e_{3}$ be the primitive idempotents of $Q V$, given by $e_{i}=$ $(1 \pm s)(1 \pm t) / 4$. There is an is omorphism $g: Q V \cong Q^{(4)}$, which we may specify by setting

$$
g^{-1}\left(x_{0}, x_{1}, x_{2}, x_{3}\right)=\sum_{i=0}^{3} x_{i} e_{i}, \quad x_{i} \in Q .
$$

Our original isomorphism (9.2) may be obtained thus:

$$
Q G \cong Q V+S \cong Q^{(4)}+S
$$

In order to determine the image of $\Lambda$ in $Q V+S$, where $\Lambda=Z G$, we use a pullback diagram. Let $Q\left[\sigma^{2}\right]$ be the group algebra of the cyclic group $\left[\sigma^{2}\right]$ generated by the element $\sigma^{2}$ in the center of $G$. Set

$$
a=\Lambda\left(\sigma^{2}+1\right), \quad b=\Lambda\left(\sigma^{2}-1\right),
$$

a pair of two-sided ideals in $\Lambda$. Since $Q G$ is $Q\left[\sigma^{2}\right]$-free, it follows at once that $a \cap \mathfrak{b}=0$.

The projection $Q G \rightarrow Q V$ induces the mappings

$$
\Lambda \rightarrow \Lambda / \mathfrak{b} \cong Z V, \quad \sigma \rightarrow s, \quad \tau \rightarrow t .
$$

On the other hand, the projection $Q G \rightarrow S$ gives

$$
\Lambda \rightarrow \Lambda / a \cong H=Z \oplus Z i \oplus Z j \oplus Z k \subset S,
$$

where $\sigma \rightarrow i, \tau \rightarrow j$. Then

$$
\Lambda(a+b) \cong \bar{Z} V \cong \bar{H}
$$

where $\bar{Z}=Z / 2 Z, \bar{H}=H / 2 H$. Let $\gamma: \bar{Z} V \cong \bar{H}$ be the above isomorphism, chosen so that $\gamma(\bar{s})=\bar{i}, \gamma(\bar{t})=\bar{j}$, where bars denote images in $\bar{Z} V$ or $\bar{H}$, depending on the context.

The pullback diagram (7.7) now becomes 


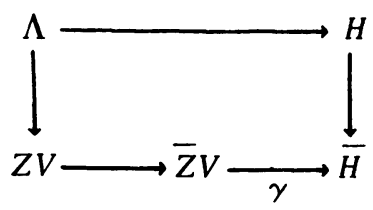

so under the isomorphism $f$, we may identify $\Lambda$ with the ring

$$
\{(v, \alpha): v \in Z V, \alpha \in H, \gamma(\bar{v})=\bar{\alpha} \text { in } \bar{H}\} .
$$

Furthermore, in terms of the is omorphism $g: Q V \cong Q^{(4)}$, it follows from (9.4) that

$$
g(Z V)=\left\{\left(x_{0}, x_{1}, x_{2}, x_{3}\right) \in Z^{(4)}: x_{0} \pm x_{1} \pm x_{2} \pm x_{3} \equiv 0(\bmod 4)\right\},
$$

where the \pm signs are chosen so that the number of minus signs is even.

Now let $H^{\prime}=H+Z \cdot \zeta, \zeta=(1+i+j+k) / 2$, so $H^{\prime}$ is a maximal order in $S$ containing $H$. As is well known, $H^{\prime}$ is a noncommutative PID and so its class group $C\left(H^{\prime}\right)$ is trivial. Set $\Lambda^{\prime}=g^{-1}\left(Z^{(4)}\right)+H^{\prime}$, a maximal $Z$-order in $Q V+S$ containing $\Lambda$; we have

$$
C\left(\Lambda^{\prime}\right) \cong C(Z)^{(4)}+C\left(H^{\prime}\right)=0 .
$$

This shows that $D(\Lambda)=C(\Lambda)$, and it remains for us to prove that $D(\Lambda)$ is of order 2. Since $S$ is a totally definite quaternion algebra, $A$ does not satisfy the Eichler condition, and so we must use the formula $D(\Lambda) \cong J\left(\Lambda^{\prime}\right) / J(\Lambda)$ in Theorem 3.11 to calculate $D(\Lambda)$.

From (9.2) or (9.3), we obtain

$$
F \cong Q^{(4)}+Q, \quad C \cong Z^{(4)}+Z,
$$

where $F$ is the center of $Q V+S, C$ the maximal $Z$-order in $F$. Since $C\left(\Lambda^{\prime}\right)=0$, it follows from $(3.11)$ that $J\left(\Lambda^{\prime}\right)$ coincides with the group $I(C, 2)$ of all $C$-ideals in $F$ prime to the rational prime 2. For brevity, let $I$ denote the group of all $Z$ ideals in $Q$ prime to 2 . Then $J\left(\Lambda^{\prime}\right)=I(C, 2) \cong I^{(5)}$.

Now let $(\nu, a) \in Q V+S$, viewed as element of $Q G$. It is readily seen that the reduced norm $N(v, \alpha) \in F$ is given by

$$
N(v, \alpha)=(g(v), N \alpha) \in Q^{(4)}+Q, \quad v \in Q V, \alpha \in S,
$$

where $N \alpha$ is the reduced norm of the quaternion $\alpha$. (If $\alpha=x_{0}+x_{1} i+x_{2} j+x_{3} k$, then $N a=\Sigma x_{i}{ }^{2}$.)

Let $U_{0}=\{1, i, j, k\}$, and let $\bar{U}_{0}$ be the image of $U_{0}$ in $u(\bar{H})$.

(9.9) Lemma. There is an epimorphism

$$
\theta: u\left(Z_{2} V\right) \times u\left(H_{2}\right) \rightarrow I^{(s)}
$$

given by

$$
\theta(v, \alpha)=Z^{(5)} \cdot N(v, \alpha)=\left(Z^{(4)} \cdot g(v), Z \cdot N \alpha\right), \quad v \in u\left(Z_{2} V\right), a \in u\left(H_{2}\right) .
$$


Furthermore, $\theta(v, \alpha) \in J(\Lambda)$ whenever $\gamma(\bar{v}) \in \bar{\alpha} \bar{U}_{0}$.

Proof. The group $I^{(5)}$ is generated by elements of the form

$$
w=\left(Z a_{0}, Z a_{1}, Z a_{2}, Z a_{3}, Z a_{4}\right), \quad a_{i} \in Z, a_{i} \text { odd. }
$$

Given such an element, we may as well adjust the $a$ 's so that

$$
a_{i} \equiv 1(\bmod 4), \quad 0 \leq i \leq 3, a_{4}>0 .
$$

By (9.7), there exists an element $v \in Z V$ such that $g(v)=\left(a_{0}, a_{1}, a_{2}, a_{3}\right)$. On the other hand, $a_{4}=N a$ for some $a \in H$, since every positive integer is a sum of four squares. Therefore we have $\theta(v, a)=w$. Since the image of $\theta$ is a group containing the generators $\{w\}$ of $I^{(5)}$, we may conclude that $\theta$ is an epimorphism, as desired.

Next, suppose that $\gamma(\bar{v})=\bar{\alpha} \bar{u}_{0}$ for some $u_{0} \in U_{0}$. Then $\left(\nu, \alpha u_{0}\right) \in \Lambda$ by (9.6), and $\theta\left(v, \alpha u_{0}\right)=\theta(v, a)$. Since $\theta\left(v, \alpha u_{0}\right) \in I(\Lambda) \subset J(\Lambda)$, the lemma is established.

Remark. Since $\theta$ is epic, and $\Lambda^{\prime} \supset Z V+H$, we conclude that $I\left(\Lambda^{\prime}\right)=I^{(5)}$. But $I\left(\Lambda^{\prime}\right) \subset J\left(\Lambda^{\prime}\right)$ obviously holds true, and therefore $J\left(\Lambda^{\prime}\right)=I^{(5)} \cong I(C, 2)$, as previously stated.

We may next deduce from Lemma 9.9 the important fact that $|D(\Lambda)| \leq 2$. We shall define an epimorphism

$$
\nu: I^{(5)} \rightarrow u(\bar{H}) / \bar{U}_{0}
$$

by setting

$$
\nu(w)=\gamma(\bar{v}) \cdot \bar{\alpha}^{-1} \cdot \bar{U}_{0}, \quad \text { where } w=\theta(v, \alpha), v \in u\left(Z_{2} V\right), \alpha \in u\left(H_{2}\right) .
$$

Let us show that $\nu(w)$ is well defined. The ideal $Z \cdot N(\alpha)$ is determined by $w$, so $a$ is determined up to a factor from $u(H)$. But $u(H)=\{ \pm 1, \pm i, \pm j, \pm k\}$, and $u(H)$ maps onto $\bar{U}_{0} \subset u(\bar{H})$, so that $\bar{\alpha}\left(\bmod \bar{U}_{0}\right)$ is uniquely determined by $w$. Secondly, $g(v) \in Z^{(4)}$ is determined up to a factor from $u\left(Z^{(4)}\right)$. The eight units $\{( \pm 1, \pm 1, \pm 1, \pm 1)\}$ in $Z^{(4)}$ are the images (under $g$ ) of the eight units $\{ \pm 1, \pm s$, $\pm t, \pm s t\}$ of $Z V$. Hence $\gamma(\bar{v})$ is determined up to a factor $\gamma\left(\bar{v}_{0}\right)$, with $v_{0} \in u(Z V)$. But $\gamma(\bar{s})=\bar{i}, \gamma(\bar{t})=\bar{j}$, so $\gamma\left(\bar{v}_{0}\right) \in \bar{U}_{0}$ for each $v_{0} \in u(Z V)$. Therefore $\gamma(\bar{v})$ is also uniquely determined in $u(\bar{H}) / \bar{U}_{0}$, and so $\nu$ is well defined. Obviously, $\nu$ is an epimorphism of multiplicative groups.

The second statement in Lemma 9.9 tells us that $\operatorname{ker} \nu \subset J(\Lambda)$. Hence we have

$$
\left[u(\bar{H}): U_{0}\right]=\left[I^{(5)}: \operatorname{ker} \nu\right]=\left[J\left(\Lambda^{\prime}\right): \operatorname{ker} \nu\right] \geq\left[J\left(\Lambda^{\prime}\right): J(\Lambda)\right]=|D(\Lambda)| .
$$

However, since $\bar{H} \cong \bar{Z} V$, we obtain $|u(\bar{H})|=|u(\bar{Z} V)|=8$. On the other hand, $\left|\bar{U}_{0}\right|=4$. Therefore, $|D(\Lambda)| \leq 2$, as claimed. 
We are now ready to use the explicit definition:

$$
J(\Lambda)=\left\{C \cdot N^{*}(x): x=\text { unit in } E\left(\Lambda_{2}+\Lambda_{2}\right)\right\},
$$

and begin by describing $E\left(\Lambda_{2}+\Lambda_{2}\right)$ as a pullback. Indeed, from (9.5) we obtain a pullback diagram

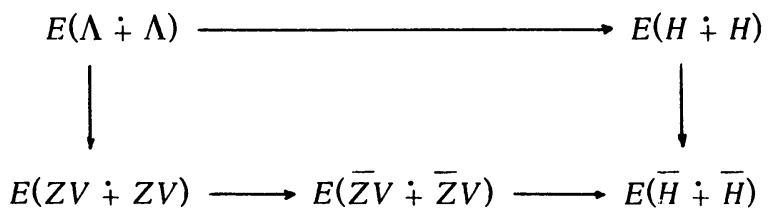

(It is obvious that this is a pullback, once we identify $E(\Lambda+\Lambda)$ with the matrix ring $(\Lambda)^{2 \times 2}$, and so on.) Localizing (9.10) at the rational prime 2 , we again obtain a pullback diagram

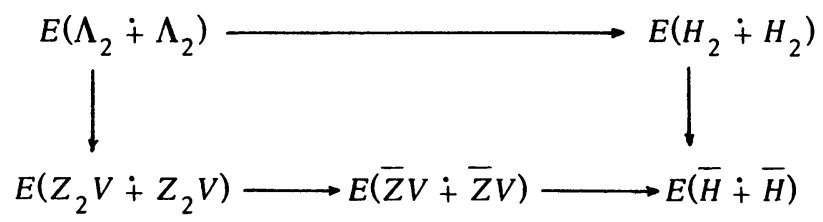

To complete the proof of Theorem 9.1, it suffices to show that the element $w=(Z, Z, Z, Z, 3 Z) \in J\left(\Lambda^{\prime}\right)$ does not lie in $J(\Lambda)$. Suppose to the contrary that $w \in J(\Lambda)$, so $w=Z^{(5)} \cdot N(x)$ for some unit $x \in E\left(\Lambda_{2}+\Lambda_{2}\right)$. By (9.11), $x$ corresponds to a pair of $2 \times 2$ matrices $X, Y$ such that

$$
\left\{\begin{array}{l}
X=\text { unit in }\left(Z_{2} V\right)^{2 \times 2}, \quad Y=\text { unit in }\left(H_{2}\right)^{2 \times 2}, \\
\gamma(\bar{X})=\bar{Y} \quad \text { as matrices over } \bar{H}, \\
Z^{(4)} \cdot N(X)=(Z, Z, Z, Z), \quad Z \cdot N(Y)=3 Z .
\end{array}\right.
$$

Since $Z_{2} V$ is commutative, the reduced norm $N(X)$ is precisely $g(\operatorname{det} X)$. But $N(X)$ is a unit in $Z^{(4)}$, so $g(\operatorname{det} X) \in u\left(Z^{(4)}\right)$; therefore (as shown in the proof of (9.9)), det $X \in u(Z V)$. Hence $\gamma(\operatorname{det} \bar{X}) \in y\{\overline{u(Z V)}\}=\bar{U}_{0}$. Therefore, we have

$$
\operatorname{det} \bar{Y} \in \bar{U}_{0^{\circ}}
$$

Let us write $Y=\left(\begin{array}{ll}a & \beta \\ \gamma & \delta\end{array}\right) \in\left(H_{2}\right)^{2 \times 2}$. Since $Y$ is invertible over $H_{2}$, it is also invertible over $\mathrm{H}_{2}^{\prime}$; but $\mathrm{H}_{2}^{\prime}$ is a local ring, so either $\alpha$ or $\gamma$ must be a unit in $H^{\prime}{ }_{2}$. It follows from Lemma 3.3 that either $\alpha$ or $\gamma$ is a unit in $H_{2}$. Suppose (without loss of generality) that $\alpha \in u\left(H_{2}\right)$. Then

$$
N(Y)=N\left(\begin{array}{ll}
\alpha & \beta \\
\gamma & \delta
\end{array}\right)=N\left(\begin{array}{cc}
\alpha & \beta \\
0 & \delta-\gamma \alpha^{-1} \beta
\end{array}\right)=N\left(\alpha \delta-\alpha \gamma \alpha^{-1} \beta\right) .
$$

The image of $a \delta-a y \alpha^{-1} \beta$ in $\bar{H}$ is just det $\bar{Y}$ (since $\bar{H}$ is commutative), and thus this image lies in $\bar{U}_{0}$ by (9.13). Therefore, 


$$
\alpha \delta-\alpha \gamma \alpha^{-1} \beta=u_{0}(1+2 t) \text { for some } t \in H_{2} \text {. }
$$

But then

$$
N\left(\alpha \delta-\alpha \gamma \alpha^{-1} \beta\right)=N\left(u_{0}\right) \cdot N(1+2 t)=1+2\left(t+t^{\prime}\right)+4 t t^{\prime},
$$

where $t^{\prime}$ is the conjugate of $t$ in $H$. This shows that $N(Y)$ is a positive rational number, congruent to $1(\bmod 4)$. The equation $Z \cdot N(Y)=3 Z$ is then impossible. This completes the proof that $J\left(\Lambda^{\prime}\right) \neq J(\Lambda)$, and that $D(\Lambda)$ has order 2 .

\section{REFERENCES}

1. H. Bass, Algebraic K-theory, Math. Lecture Note Series, Benjamin, New York, 1968. MR $40 \# 2736$.

2. N. Bourbaki, Éléments de mathématique. XXIII. Part I. Les structures fondamentales de l'analyse. Livre II: Algèbre. Chap. 8, Actualités Sci. Indust., no. 1261, Hermann, Paris, 1958. MR $20 \# 4576$.

3. C. W. Curtis and I. Reiner, Representation theory of finite groups and associative algebras, Pure and Appl. Math., vol. XI, Interscience, New York, 1962; 2nd ed., 1966. MR $26 \# 2519$.

4. M. Deuring, Algebren, Springer-Verlag, Berlin, 1935; Zweite korrigierte Auflage, Ergebnisse der Mathematik und ihrer Grenzgebiete, Band 41, 1968. MR 37 \#4106.

5. M. Eichler, Allgemeine Kongruenzklasseneinteilungen der Ideale einfachen Algebren über algebraischen Zahlkörpern und ihre L-Reihen, J. Reine Angew. Math. 179 (1938), 227-251.

6. W. Feit, Characters of finite groups, Math. Lecture Note Series, Benjamin, New York, 1967. MR $36 \# 2715$.

7. A. Fröhlich, Ideals in an extension field as modules over the algebraic integers in a finite number field, Math. Z. 74 (1960), 29-38. MR $22 \sharp 4708$.

8. matika 16 (1969), 143-152. MR 41 \#5512.

9. H. Jacobinski, Genera and decompositions of lattices over orders, Acta. Math. 121 (1968), 1-29. MR $40 \# 4294$.

10. M. P. Lee, Integral representations of dihedral groups of order $2 p$, Trans. Amer. Math. Soc. 110 (1964), 213-231. MR $28 \# 139$.

11. I. Reiner, A survey of integral representation theory, Bull. Amer. Math. Soc. 76 (1970), 159-227. MR $40 \# 7302$.

12. K. W. Roggenkamp and V. H. Dyson, Lattices over orders. I, II, Lecture Notes in Math., nos. 115, 142, Springer-Verlag, Berlin and New York, 1970.

13. R. G. Swan, Induced representations and projective modules, Ann. of Math. (2) 71 (1960), 552-578. MR $25 \# 2131$.

14. - The Grothendieck ring of a finite group, Topology 2 (1963), 85-110. MR $27 \# 3683$.

15. R. G. Swan and E. G. Evans, K-theory of finite groups and orders, Lecture Notes in Math., no. 149, Springer-Verlag, Berlin and New York, 1970.

16. S. Ullom, A note on the classgroup of integral group rings of some cyclic groups, Mathematika 17 (1970), 79-81. MR $42 \sharp 4650$.

17. A. Fröhlich, The Picard group (to appear).

18. I. Reiner and S. Ullom, A Mayer-Vietoris sequence for class groups, J. Algebra (to appear).

19. A. Fröhlich, I. Reiner and S. Ullom, Picard groups and class groups (to appear).

20. J. Martinet, Modules sur l'algèbre du groupe quaternionien, Ann. Sci. École Norm. Sup. 4 (1971), 399-408.

DEPARTMENT OF MATHEMATICS, UNIVERSITY OF ILLINOIS AT URBANA-CHAMPAIGN, URBANA, ILLINOIS 61801 